\title{
Increasing Returns and Optimal Oscillating Labor Supply*
}

\author{
William D. Dupor \\ The Wharton School \\ University of Pennsylvania \\ 2300 Steinberg Hall-Dietrich Hall \\ Philadelphia, PA 19104 \\ (215) 8987634 \\ Dupor@wharton.upenn.edu
}

\author{
Andreas Lehnert \\ Board of Governors of the \\ Federal Reserve System \\ Mail Stop 93 \\ Washington, DC 20551 \\ (202) 452-3325 \\ Andreas . Lehnert@FRB . GOV
}

This Version: April 2002

Last Revised: April 17, 2002

*Dupor thanks the Hoover Institution for its support and hospitality. The views expressed in the paper are ours alone and do not necessarily reflect those of the Board of Governors of the Federal Reserve System or its staff. The authors would like to thank Larry Christiano, Joao Gomes, Robert Hall, Ken Judd, Kevin Lansing, Per Krusell and Robert Shimer for helpful conversations. This paper has additionally benefitted from the comments of seminar participants at the 2001 NBER summer institute and the Federal Reserve Board. 



\title{
Increasing Returns and Optimal Oscillating Labor Supply
}

\begin{abstract}
Models featuring increasing returns to scale in at least one factor of production have been used to study two separate phenomena: (1) multiplicity of self-fulfilling rational expectations equilibria (i.e. sunspots), and (2) production schedules that optimally feature bunching. We show in a continuous-time model with increasing returns to labor (IRL) that if the economy features multiple competitive equilibria, the optimal path of investment, employment and consumption cannot be constant, or even smoothly-varying. Any macroeconomic policies that shielded the economy from sunspot fluctuations would necessarily not be optimal. We then characterize the optimal allocation (the solution to the planner's problem) in a discrete time version of the model. We find that the optimal investment, employment and consumption policies under increasing returns can feature (1) discontinuous jumps, (2) endogenous cycles (with time-varying cycle limits) and (3) stochastic controls (lotteries). Our discrete-time model is very close to that studied by Christian and Harrison (1999); they, however, find that fluctuations are not optimal. We show that this difference is driven by their assumption that production is linear in capital.
\end{abstract}

Journal of Economic Literature classification numbers: E32, E33, C61, C62, D62 Keywords: Increasing returns, externalities, fluctuations, lotteries 



\section{Introduction}

Economists routinely use dynamic models which feature many competitive equilibria to study macroeconomic cycles. In these models, severe swings in output and employment can follow phenomena that have no fundamental economic significance (usually referred to as sunspots). Research has naturally focused the existence and nature of endogenous equilibrium cycles, as well as on designing fiscal and monetary policies to rule them out. However, despite their ultimate goal of formulating macroeconomic policies, very few papers have explicitly studied the optimal paths of employment and investment. In the context of a popular multipleequilibrium model, we show that the necessary conditions for multiple equilibria are sufficient to preclude a smooth optimal path for investment and labor. Any policy that successfully eliminated equilibrium cycles would thus necessarily not be optimal.

The model we study involves increasing returns to labor to generate multiple equilibria. This is a popular choice in the literature, and leads to a natural interpretation of multiple equilibria. Intuitively, consider the simple case of a static model in which many identical households must decide how hard to work. The production function features increasing social returns but constant private returns to labor. If households expect a high wage, they supply high labor to the market. If they expect a low wage, they supply less labor to the market. Because the marginal product of labor is increasing in aggregate labor supply, in a symmetric equilibrium households' expectations are confirmed. Thus either set of expectations and 
corresponding actions satisfy the conditions for an equilibrium.

More sophisticated dynamic models exploring the connections among increasing returns, expectations and equilibrium indeterminacy include Kiyotaki’s (1988) study of equilibrium investment and government policy; Murphy, Vishny and Shleifer's (1989a) argument that externalities must play a key role in business cycles; Baxter and King's (1991) study of externalities and business cycles; Benhabib and Farmer's (1994, 1996) and Farmer and Guo's (1994, 1995) studies of “animal spirits" models; Boldrin and Rustichini's (1994) study of two-sector models with externalities and Subrahmanyam and Titman's (1999) study of the role of equity prices as an equilibrium selection mechanism.

A different literature has pursued the implications of increasing returns on optimal production policies. In a spatial context, for example, economists have studied how small initial differences across locations can powerfully affect the long-run distribution of industries. ${ }^{1}$ In the same way that production bunching across space might be optimal under increasing returns, in a dynamic setting, it may be optimal to bunch production across time. That is, a social planner, faced with increasing returns to labor, might optimally direct households to work hard for brief periods of time and then relax. During the spurts of labor, households accumulate a large capital stock which they then consume during the relatively quiet time. If households are sufficiently willing to substitute leisure between periods, and have some means of storing output from one period to the next, they will take

\footnotetext{
${ }^{1}$ We can at best merely scratch the surface of the large literature on increasing returns, spillovers and the development of countries and cities; see, e.g. Murphy, Vishny, and Shleifer (1989a), Lucas (1993) or Krugman (1999).
} 
advantage of increasing returns to labor and work hard when labor effort is already high, because the marginal product of labor is increasing. Murphy, Shleifer and Vishny (1989a, p. 250) recognize this point in a durable consumption goods model with increasing returns to labor, but without capital: "[i]t is efficient for this industry to produce at capacity some of the time and to rest other times, rather than to always produce at a constant output level." A complementary, data-driven view is that business cycles are largely driven by inventory cycles. For example, inventory adjustments account for 87 percent of the drop in GNP during the average post-war U.S. recession (Blinder and Maccini, 1991). Further, there has been increasing interest recently in spillovers, or externalities in labor productivity, as a part of the "new" economy. Thus the type of cycle driven by labor externalities is not utterly unlike the kind of business cycle that characterizes the U.S. economy.

Despite this natural intuition and a decade's worth of theoretical and applied interest, there have been few efforts to characterize optimal resource allocations under increasing returns to labor in the neoclassical growth model. ${ }^{2}$ Instead, most work has focused on designing policies to eliminate equilibrium cycles in the model. Put another way, research has concentrated on the conditions under which competitive equilibria are unique, generally ignoring the optimal plan. For example, Guo and Lansing (1998) show that a progressive tax can render otherwise indeterminate equilibria locally unique, shielding the economy from sunspot fluctuations.

In this paper, we characterize the connection between equilibrium indetermi-

\footnotetext{
${ }^{2}$ We will consider one exception, Christiano and Harrison (1999), in great detail below.
} 
nacy and optimal cycles in the neoclassical growth model with increasing returns to labor. We begin by finding the equilibria of a continuous time neoclassical growth model featuring increasing social returns to labor but diminishing social returns to capital. Following Benhabib and Farmer (1994), we show that increasing returns to labor in production is a necessary condition for local equilibrium indeterminacy of the steady-state equilibrium. We establish that this necessary condition for indeterminacy is sufficient to ensure that the optimal paths of labor and investment cannot be constant. In fact, the optimal path cannot even be smoothly varying because we show that, if it exists, the path is not a piecewise continuous function of time.

Instead of attempting to characterize the optimal allocation in this case (for example, by imposing adjustment costs or recasting the problem as one of choosing a probability distribution over effort levels) we move on to study the discrete-time version of the model. In discrete-as opposed to continuous-time, the planner must choose one level for effort and maintain it throughout the period. We find that the planner's optimal labor policy (as a function of beginning of period capital) generally features a sharp drop at a critical capital level, so that when the economy is richer than the critical point, optimal labor effort drops to zero. At this critical point, households consume some or all of the existing capital stock. If this critical point lies along the optimal dynamic capital accumulation path, the economy will feature oscillating labor supply and the capital stock will cycle over time. Moreover, we find that, although the planner could completely smooth consumption over time, he chooses not to. Intuitively, the shadow price of consumption varies 
with effort and investment, leading the planner to trade off the utility benefits and the technological costs of smoothing.

Even aside from the single discontinuous drop down in effort at the critical level of capital, the optimal labor effort schedule can feature several separate discontinuous jumps up at lower levels of capital. In essence, the increasing returns to labor cause the household's utility as a function of investment to feature several local maxima. As beginning of period capital increases, the global maximum can hop among these various local maxima, and their associated investment policies. Thus not only is it possible for the optimal path of labor to cycle over time, the limits of the cycle can, as a result of these sharp jumps up, also cycle over time.

Finally, we show that, under certain circumstances, the planner prefers using stochastic policies or capital gambles. This makes sense; our results are driven by the fact that the planner's static return function features a sharp kink at the point where optimal labor effort drops from a positive level to zero. Faced with an initial capital stock within the kinked region, the planner would prefer to gamble over a high capital stock and a low capital stock rather than use the inherited capital stock. Note however that the planner does not always want to use lotteries; by cleverly choosing non-stochastic policies, the planner will, under certain parameters, be able to come arbitrarily close to the solution with lotteries.

The discrete-time version of our model is close to that studied by Christiano and Harrison (CH, 1999), who also characterize the optimal plan. They, however, find that labor supply is smooth in the capital stock, that production cycles are not optimal and, as a result, that the planner would not use stochastic controls if 
offered them. The key difference between our model and theirs is the social return to capital. We assume that social returns to capital are decreasing; in contrast, they assume that social returns to capital are constant. In essence, in our model, with decreasing returns to capital, the planner finds it optimal to work very hard today in order to build up a large capital stock. In the next period, although the marginal product of labor will be higher (because the capital stock is higher) it will be less than linearly higher, as in CH's model. If the planner in CH's model attempted to replicate the optimal plan from our model by working hard today, he would find that, tomorrow, labor's productivity would be linearly higher than today's. But labor's productivity is also the opportunity cost of taking vacations, and the planner would find a vacation too expensive following a burst of effort. In our model, labor's product does not increase one-for-one with the capital stock as it does in their model. Beyond some critical point, labor's product dips enough so that the planner prefers to take a vacation and consume leisure.

The plan of our paper is as follows: in section 2, we develop the implications of equilibrium indeterminacy for the nature of optimal labor supply paths in the continuous time Benhabib-Farmer model. In section 3, we study optimal resource allocations in a discrete-time model. We characterize the planner's static return function and develop a simplified finite-horizon model to provide some intuition for our results. We also provide results linking increasing returns to scale in labor to stochastic controls. In section 4 we numerically characterize the discrete-time planner's problem. We choose a benchmark set of parameters and then vary all the parameters of interest around the benchmark. Cycles are more likely to be 
optimal with large increasing returns to labor, slow capital depreciation and a low utility time discount rate. We also compute the optimal policies with lotteries. Section 5 briefly concludes. All proofs of our theorems and lemmas are relegated to appendix A. In appendix B we provide a comparison with the exact ChristianoHarrison specification.

\section{Increasing Returns in Continuous Time}

\subsection{Increasing Returns to Labor Are Necessary for Local Equi- librium Indeterminacy}

Consider the neoclassical growth model with external returns to scale in production. A representative household maximizes the present discounted valued of a log-linear felicity function defined in terms of flows of consumption $(c)$ and labor effort $(n)$. The discount rate is $\rho>0$, so the maximization problem becomes:

$$
\int_{0}^{\infty} e^{-\rho t}[\log (c)-\phi n] d t,
$$

subject to:

$$
\dot{k}=(r-\delta) k+w n+\pi-c,
$$

and an initial condition $k(0)=k_{0}>0$. As usual, $k$ denotes the level of aggregate capital. Here, the scalar parameters $\rho, \delta$, and $\phi$ are all strictly positive and the 
household takes the real interest rate $r$, wage $w$ and profits $\pi$ as given.

Firm-level production is given by:

$$
y=k^{a} n^{g}\left[\bar{k}^{\alpha-a} \bar{n}^{1+\gamma-g}\right]
$$

where the scalar parameters $a, g, \alpha$, and $\gamma$ satisfy $a, g>0,1>\alpha>a, 1+\gamma>g$ and $a+g=1$. Here, $k, n$ denote firm-level capital and labor usage, while $\bar{k}, \bar{n}$ are the economy-wide averages. The parameters $a, g$ govern the private returns to capital and labor (as opposed to the social returns). The social (total) production function is given by:

$$
y=k^{\alpha} n^{1+\gamma}
$$

Firms maximize profits, taking as given factor prices and economy-wide average input use. Profit-maximization implies:

$$
y / k=r / a, \text { and: }
$$

$$
y / n=w / g \text {. }
$$

By assuming constant private returns to scale $(a+g=1)$, firm profits equal zero in a competitive equilibrium. 
The necessary conditions for consumer optimization are:

$$
\begin{aligned}
c & =\frac{w}{\phi}, \\
\frac{\dot{c}}{c} & =r-\rho-\delta .
\end{aligned}
$$

Also, the paths of capital and consumption must satisfy a transversality condition:

$$
\lim _{T \rightarrow \infty} e^{-\rho T} \frac{k(T)}{c(T)}=0
$$

For the remainder of the analysis, we study symmetric equilibria, in which the aggregate quantities of capital and labor, $\bar{k}$ and $\bar{n}$, are given by the representative household's optimal choice when it takes $\bar{k}$ and $\bar{n}$ as given.

Use the profit-maximization conditions, equations (2.3) and (2.4), to eliminate factor prices $r$ and $w$ from the necessary conditions from the consumer's problem, equations (2.5) and (2.6):

$$
\begin{aligned}
c & =\frac{g}{\phi} \frac{y}{n}, \\
\frac{\dot{c}}{c} & =a \frac{y}{k}-\rho-\delta .
\end{aligned}
$$

After substituting out factor prices, the law of motion for capital, equation (2.1), becomes:

$$
\dot{k}=y-\delta k-c .
$$


Equations (2.2), (2.8), (2.9), (2.10), along with the transversality condition (2.7), describe the equilibrium dynamics of the economy.

We can use the production function, equation (2.2), and the relationship of consumption to the output-labor ratio, equation (2.8), to substitute output $y$ and labor $n$ out of the differential equations governing the evolution of consumption (2.9) and capital (2.10). Begin by eliminating labor effort from output, so that:

$$
y=k^{-\alpha / \gamma}\left(\frac{\phi}{g} c\right)^{(1+\gamma) / \gamma} .
$$

Thus equilibrium consumption and capital jointly evolve according to the system of equations:

$$
\begin{aligned}
& \frac{\dot{c}}{c}=a\left(\frac{\phi}{g}\right)^{\frac{1+\gamma}{\gamma}} k^{-\frac{\alpha+\gamma}{\gamma}} c^{\frac{1+\gamma}{\gamma}}-\rho-\delta, \\
& \dot{k}=\left(\frac{\phi}{g}\right)^{\frac{1+\gamma}{\gamma}} k^{-\frac{\alpha}{\gamma}} c^{\frac{1+\gamma}{\gamma}}-\delta k-c .
\end{aligned}
$$

We next turn to determining the nature of equilibria generated by this system.

We begin by $\log$-linearizing the system. First, define $C=\log (c)$ and $K=$ $\log (k)$. The dynamic system, equations (2.11) and (2.12), can be rewritten as the autonomous differential equations:

$$
\begin{aligned}
& \dot{C}=a \exp \left(D_{0}-D_{1} K+D_{2} C\right)-\rho-\delta \\
& \dot{K}=\exp \left(D_{0}-D_{1} K+D_{2} C\right)-\delta-\exp (C-K) .
\end{aligned}
$$


For convenience, we defined the constants $D_{0}, D_{1}$ and $D_{2}$ as:

$$
D_{0}=\frac{1+\gamma}{\gamma} \log \left(\frac{\phi}{g}\right), D_{1}=\frac{\alpha+\gamma}{\gamma} \text {, and } D_{2}=\frac{1+\gamma}{\gamma}
$$

Every path for capital and consumption that satisfies the transversality condition, equation (2.7), the system of equations (2.13) and (2.14), and the initial condition for capital is a perfect foresight equilibrium. It is straightforward to show that there is a unique steady-state equilibrium. Define $K^{s s}$ and $C^{s s}$ as the equilibrium steady-state levels of capital and consumption. We exploit the following two steady-state implications of equations (2.13) and (2.14):

$$
\begin{aligned}
\exp \left(D_{0}-D_{1} K^{s s}+D_{2} C^{s s}\right) & =\frac{\rho+\delta}{a}, \\
\exp \left(C^{s s}-K^{s s}\right) & =\frac{\rho+\delta}{a}-\delta .
\end{aligned}
$$

We next wish to study the local determinacy properties of the steady-state equilibrium. Linearizing the system equilibrium equations written in terms of $\log$ capital and log consumption, equations (2.13) and (2.14), we have:

$$
\left[\begin{array}{c}
\dot{C} \\
\dot{K}
\end{array}\right]=\left[\begin{array}{cc}
(\rho+\delta)\left(\frac{1+\gamma}{\gamma}\right), & -(\rho+\delta)\left(1+\frac{\alpha}{\gamma}\right) \\
\delta+\frac{\rho+\delta}{a \gamma}, & -\left[\delta+\frac{\alpha(\rho+\delta)}{\gamma a}\right]
\end{array}\right]\left[\begin{array}{c}
C-C^{s s} \\
K-K^{s s}
\end{array}\right]
$$

Let $A$ denote the square matrix in (2.15). We are ready to present a result concerning indeterminacy which was first worked out by Benhabib and Farmer (1994). 
Theorem 1 The $\left(C^{s s}, K^{s s}\right)$ equilibrium is locally indeterminate if and only if:

1. Returns to labor are increasing: $\gamma>0$,

2. The trace of the transition matrix $A$ is negative, or:

$$
\delta>(\rho+\delta)[(1+\gamma) a-\alpha] /(\gamma \alpha)
$$

Theorem 1 holds that a necessary condition for equilibrium indeterminacy is social returns to labor that are increasing in scale (in other words, that $\gamma>0$ ). This is intuitive; as Benhabib and Farmer (1994) explain, when $\gamma>0$, aggregate labor demand is upward-sloping and, in fact, steeper than aggregate labor supply at high levels of labor. Further, when $\gamma>0$ it is also possible that the equilibrium is a source, so that any small deviation from the steady-state will result in an infinite expansion. For example, we assumed that the private returns to capital, $a$, are less than the social returns, $\alpha$. If instead we assume that $a=\alpha$, the trace of the transition matrix $A$ becomes $\operatorname{tr}(A)=\rho>0$ (violating condition 2 in theorem 1). As a result, without a wedge between social and private returns to capital (even though both are decreasing in scale), the equilibrium will be locally explosive.

Requiring a wedge between the social and private returns to capital is the same as assuming that at least some of capital's product is external to the firm. These capital externalities militate against explosiveness of the equilibrium dynamics; they bring the system back to the steady state after an expectations shock. Imagine 
the economy begins with capital at the steady-state level. At time zero, households develop expectations that their neighbors will work harder than the steady-state effort level, both in the instant $t=0$, and for at least a short while thereafter. Associated with this expectation of the path of average labor, households also expect a gradual rise and then decline in average capital holding. Expecting higher shortrun (labor and capital) productivity, households increase own labor supply, which validates their expectations of higher average labor input, and increase savings to smooth consumption. The increased savings, in addition, augments the marginal productivity of labor in the future. If the household fully internalized the returns to capital $(a=\alpha)$, its future path of capital would continue to increase. However, because of the wedge in capital's product, the average household persistently undersaves, allowing capital to decline.

\subsection{Increasing Returns to Labor Are Sufficient to Preclude a Smooth Optimal Path for Labor}

In addition to being a necessary condition for equilibrium indeterminacy, increasing returns also has implications for the Pareto optimal resource allocation in the Benhabib-Farmer model. To study the optimal allocation, we use some of the theoretical apparatus of Pontryagin's Maximum Principle (PMP). We briefly review the required results before turning our attention to the planner's problem. 
Consider the following infinite horizon problem (or IHP):

$$
\max _{u(t), 0 \leq t} \int_{0}^{\infty} e^{-\rho t} f(x(t), u(t)) d t
$$

where $x(t)$ is a scalar function which is free as $t \rightarrow \infty$ and has an initial value given by $x(0)=x_{0}$. The controls $u(t) \in U \subset \mathbb{R}^{m}$ influence the evolution of the state $x$ as:

$$
\dot{x}(t)=g(x(t), u(t)) \text {. }
$$

We will require that under all admissible trajectories of the controls $u(t) \in U$, the integral in the objective $(2.16)$ converges. ${ }^{3}$

The current value Hamiltonian for the infinite horizon problem is:

$$
H^{c}(x, u, \lambda)=\lambda_{0} f(x, u)+\lambda g(x, u)
$$

We now appeal to the results in Pontryagin et al (1964, theorems 1 and 2).

Definition 1 (The Pontryagin Maximum Principle) If $x^{\star}(t)$ and $u^{\star}(t)$ are admissible paths for the IHP and are both piecewise optimal, then there exists a constant $\lambda_{0}$ and a continuous scalar function $\lambda(t)$ such that for all $t \geq 0$ :

1. $\lambda_{0}=0$ or 1 , and $\left(\lambda_{0}, \lambda(t)\right)$ is never $(0,0)$.

\footnotetext{
${ }^{3}$ The gimlet-eyed reader will have noticed that we have also assumed that the objective $f$ and the control $g$ are autonomous functions of the controls and the state. This is merely to tie this more general discussion to our specific problem. It is otherwise innocuous.
} 
2. For all controls $u$ in $U$ :

$$
H^{c}\left(x^{\star}(t), u, \lambda(t)\right) \leq H^{c}\left(x^{\star}(t), u^{\star}(t), \lambda(t)\right) .
$$

3. At points where $u^{\star}(t)$ is continuous:

$$
\dot{\lambda}-\rho \lambda=-\frac{\partial}{\partial x} H^{c}\left(x^{\star}, u^{\star}, \lambda\right) .
$$

We next turn to the application of the PMP for the particular IHP first studied by Benhabib and Farmer.

Definition 2 (Social Planner's Problem) The planner maximizes:

$$
\int_{0}^{\infty} e^{-\rho t}[\log (c(t))-\phi n(t)] d t
$$

where the control vector $[c(t), n(t)]^{\prime} \geq 0$ for all $t$. The capital stock $k(t)$ (the single state variable) is free as $t \rightarrow \infty$ and the initial capital stock is given by $k(0)=k_{0}>0$. The dynamics of the capital stock are governed by the law of motion:

$$
\dot{k}(t)=k(t)^{\alpha} n(t)^{1+\gamma}-c(t)-\delta k(t) .
$$

We assume, as in the decentralized model, that $0<\alpha<1, \delta, \phi>0$, and $\gamma \geq$ $\alpha-1$. 
Theorem 2 If $\gamma>0$, there does not exist a piecewise continuous optimal path for consumption and labor for the social planner's problem from definition 2 above.

The intuition for theorem 2 is straightforward: condition (2) of the PMP holds that for a given level and shadow value of the state variable, $k^{\star}(t)$ and $\lambda(t)$, the controls must be chosen to maximize the current value Hamiltonian. However, at each instant, the social planner faces increasing returns to labor but linear disutility of work. For a given productivity of labor $\left(k^{\star}\right)^{\alpha}$ and shadow value of consumption $\lambda$, increasing returns to labor implies that there is no interior solution to the social planning problem at each instant. Indeed, every piecewise continuous path for labor and investment is dominated by some other piecewise continuous path. We conjecture that the optimal plan may involve a chattering solution, or arbitrarily frequent discontinuous jumps in the labor and investment time paths. Romer (1986) points out that such solutions have little economic meaning, and advises putting more structure on problems with this feature.

The particular structure that we adopt is to move our analysis to discrete time. This strategy has two benefits. First, moving to discrete time provides an elegant circumvention of the chattering problem. In essence, we are requiring the planner to commit to a particular effort level for the length of period, in the spirit of Stokey (1981). This forces the optimal plan to be a piecewise continuous function of time. Second, given that our results differ from those of Christiano and Harrison (1999), moving to discrete time will permit a more direct comparison. 


\section{Increasing Returns in Discrete Time}

In this section we describe the discrete time analog to the continuous time social planner's problem from definition 2 above. We show that the static part of the planner's objective function features a distinct kink, that the planner's choices of investment and labor policies jump down at this kink and, further, that they can jump up to the left of this kink. We also show that the planner would use certain types of stochastic controls (that is, lotteries) if permitted.

One reason that we study a discrete-time model is to make our results comparable with Christiano and Harrison, who study a similar model but find that the Pareto optimal policy involves a constant labor supply. Our model differs from theirs in three ways. The most important difference is that we assume diminishing-as opposed to constant-returns to scale in capital. The other differences are the functional forms of preferences and technology; in particular, we assume linear disutility of labor and returns to labor of $n^{1+\gamma}$ as opposed to $n^{2}$. In appendix B we solve the exact Christiano-Harrison model with diminishing returns to capital and find that it produces endogenous optimal cycles. Finally, it is worth noting that Christiano and Harrison explicitly recognized the important role that constant returns to capital might have in their model: "We have shown that this [optimality of constant labor input] is so under a particular homogeneity assumption on the resource constraint. But, standard models do not satisfy this condition (1999, p. 24)." 


\subsection{Model}

At the beginning of period $t$, the social planner faces the problem of choosing streams of labor and consumption to maximize the household's utility:

$$
v_{t}\left(k_{t}\right)=\max _{\left\{c_{t+i}, n_{t+i}\right\}_{i=0}^{\infty}} \sum_{i=0}^{\infty} \beta^{i}\left[\log \left(c_{t+i}\right)-\phi n_{t+i}\right], 0<\beta<1 .
$$

The economy-wide technology transforms period- $t$ labor $n_{t}$ and capital $k_{t}$ into output as:

$$
Y\left(k_{t}, n_{t}\right)=k_{t}^{\alpha} n_{t}^{1+\gamma} .
$$

We will generally take $0 \leq \alpha<1$ and $\gamma \geq 0$. However, note that if $-1<\gamma \leq-\alpha$ the production function is Cobb-Douglas with decreasing (or at best constant) returns to scale in capital and labor.

The capital stock evolves as:

$$
k_{t+1}=k_{t}^{\alpha} n_{t}^{1+\gamma}+(1-\delta) k_{t}-c_{t} .
$$

In each period $t$, the planner maximizes the continuation utility (3.1) subject to the capital evolution equation (3.3).

We will find it more convenient not to work with the planner's sequence problem, equation (3.1) above, but rather with the recursive version of the planner's 
problem. Written recursively, the planner's problem becomes:

$$
\begin{gathered}
V(k)=\max _{k^{\prime}, c, n}\left\{\log (c)-\phi n+\beta V\left(k^{\prime}\right)\right\}, \text { subject to: } \\
\qquad k^{\prime}=k^{\alpha} n^{1+\gamma}+(1-\delta) k-c, \\
\text { and } k, k^{\prime} \in \mathbf{K}=\left[k_{\min }, k_{\max }\right], k_{\min }>0, k_{\max }<\infty .
\end{gathered}
$$

Define $\mathbb{B}(\mathbf{K})$ as a space of bounded continuous functions $f: \mathbf{K} \rightarrow \mathbf{R}$ (where $\mathbf{R} \subset \mathbb{R})$. Define the functional operator $\mathcal{T}: \mathbb{B}(\mathbf{K}) \rightarrow \mathbb{B}(\mathbf{K})$ as:

$$
(\mathcal{T} f)(k)=\max _{n, k^{\prime}} \log \left(k^{\alpha} n^{1+\gamma}+(1-\delta) k-k^{\prime}\right)-\phi n+\beta f\left(k^{\prime}\right) .
$$

In section A.5 below we establish that the solution to the recursive problem, the value function $V(k)$, is the same as the solution to the sequence problem, $v_{t}(k)$ for all periods $t$. Moreover, we show that the operator $\mathcal{T}$ satisfies the conditions for a contraction mapping. Thus unlike the continuous time version of our model, the discrete time version will at least have some kind of solution that we have a hope of finding with standard techniques, even under increasing returns to scale.

\subsection{The Static Return Function}

We now show that we can decompose the recursive problem, equation 3.4 above, into a static piece and a dynamic piece; moreover, we show that the static piece is purely a function of initial capital and the choice of continuation capital. We analyse this static return function and show that it features a distinct set of kinks 
at critical values of the current capital stock. In one region, the planner will work hard; beyond the kinks, though, the planner will set labor effort to zero.

For a given level of capital today, $k$, imagine first fixing the choice of capital tomorrow, $k^{\prime}$, and then deciding how much to work today, $n$. This will generate a one-period return function $X\left(k, k^{\prime} ; n\right)$ defined as:

$$
X\left(k, k^{\prime} ; n\right) \equiv \log \left(k^{\alpha} n^{1+\gamma}+(1-\delta) k-k^{\prime}\right)-\phi n .
$$

By bringing out the $k^{\alpha}$ term, this may be rewritten as:

$$
X\left(k, k^{\prime} ; n\right)=\alpha \log (k)+\log \left(n^{1+\gamma}+\frac{(1-\delta) k-k^{\prime}}{k^{\alpha}}\right)-\phi n .
$$

Define:

$$
F\left(k, k^{\prime} ; n\right)=\log \left(n^{1+\gamma}+h\right)-\phi n, \text { where } h \equiv \frac{(1-\delta) k-k^{\prime}}{k^{\alpha}} .
$$

Note that $h$ can take on large negative or positive values. The planner's goal, given $k, k^{\prime}$ is to choose effort $n$ to maximize $F\left(k, k^{\prime} ; n\right)$ and with it the static return function $X$. Thus $F\left(k, k^{\prime}\right)$ is:

$$
F\left(k, k^{\prime}\right) \equiv \max _{n}\left\{\log \left(n^{1+\gamma}+h\right)-\phi n\right\}
$$

Define $n^{\star}\left(k, k^{\prime}\right)$ as the optimal effort policy from this problem. The recursive 
problem (3.4) now becomes:

$$
V(k)=\max _{k^{\prime}}\left\{\alpha \log (k)+F\left(k, k^{\prime}\right)+\beta V\left(k^{\prime}\right)\right\}
$$

We will now characterize $F$, with the goal of characterizing, to the extent possible, $V$. We begin with a technical result:

Lemma 1 The functions $F\left(k, k^{\prime}\right)$ and $n^{\star}\left(k, k^{\prime}\right)$ defined in equation (3.8) above satisfy:

1. For all $k, k^{\prime}$ in $\mathbf{K} \times \mathbf{K}, F$ is bounded and continuous.

2. For all $k, k^{\prime}$ in $\mathbf{K} \times \mathbf{K}, n^{\star}$ is bounded.

We next turn to characterizing the optimal effort policy:

Lemma 2 The optimal effort policy, $n^{\star}\left(k, k^{\prime}\right)$ satisfies:

1. If $\gamma<0, n^{\star}\left(k, k^{\prime}\right)>0$ all $k, k^{\prime}$ in $\mathbf{K} \times \mathbf{K}$.

2. If $\gamma>0$ there exists a scalar $h_{\max }>0$ such that for all $k, k^{\prime}$ in $\mathbf{K} \times \mathbf{K}$ satisfying $k^{\prime}<-h_{\max } k^{\alpha}+(1-\delta) k$, the optimal effort is zero, $n^{\star}\left(k, k^{\prime}\right)=0$.

3. If $\gamma>0$ and labor effort is greater than zero, $n^{\star}>0$, then labor effort must be above a positive threshold level, $n^{\star} \geq \gamma / \phi>0$.

Lemma 2 essentially states that increasing returns to scale in labor (that is, $\gamma>$ 0 ) is a necessary condition for the optimality of labor cycles. If the optimal choice of labor is smoothly varying the problem collapses to the standard neoclassical 
growth model. In the presence of increasing returns, however, the optimal choice of labor features a discrete jump down. This will induce a set of kinks in the static return function $F\left(k, k^{\prime}\right)$; everywhere that $n>0, F$ will have a relatively shallow slope in $k$ (positive) and $k^{\prime}$ (negative), because the planner is also adjusting labor. In the region where $n=0$, by contrast, the slope will be relatively steeper, because consumption will be $(1-\delta) k-k^{\prime}$.

In figures 1 and 2 below we plot the region in which the planner works hard and that in which he does not, and we also plot the static return function $F$ for several values of $\gamma$. Notice the distinct kink in $F$ for even small values of $\gamma>0$; this kink disappears in the case where $\gamma<0$, that is, diminishing returns to scale in labor.

Lemma 2 also shows that if $\gamma>0$ there will some region of low, but nonzero, labor effort that the planner will simply never choose. As he approaches this region, the planner jumps down to zero. This also underlies the kinks in figure 2.

\subsection{Finite Horizon}

In order to understand how the infinite-horizon planner's problem behaves, $V(k)$, it is useful to consider the solution to the finite horizon case. In particular, using a finite horizon version of the planner's problem, we show that this model can exhibit other unusual features, even beyond oscillating labor supply. As we saw in lemma 2 above, the planner never chooses a non-zero labor level that is also less than $\gamma / \phi$. Thus when $\gamma>0$, the planner's labor policy function can suddenly

jump down to zero. This behavior underlies the oscillations in labor and capital 


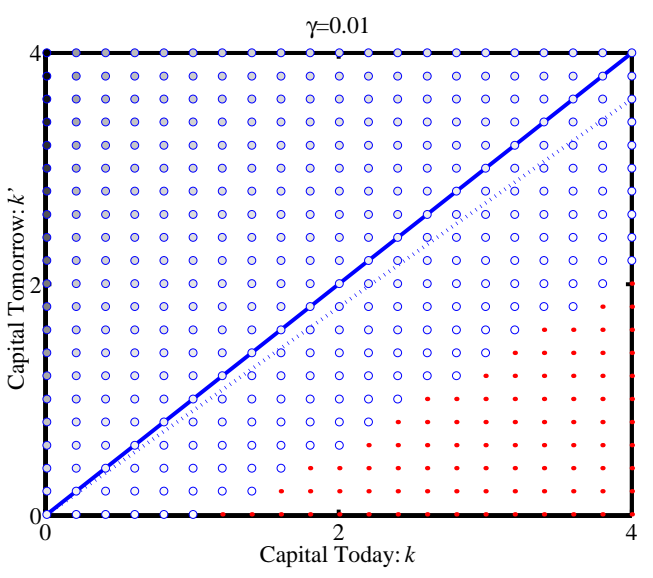

Figure 1: Planner's work decision for $k, k^{\prime}$; the circles give points at which the planner works hard, the dots where he does not. The dashed line gives (1$\delta) k$.

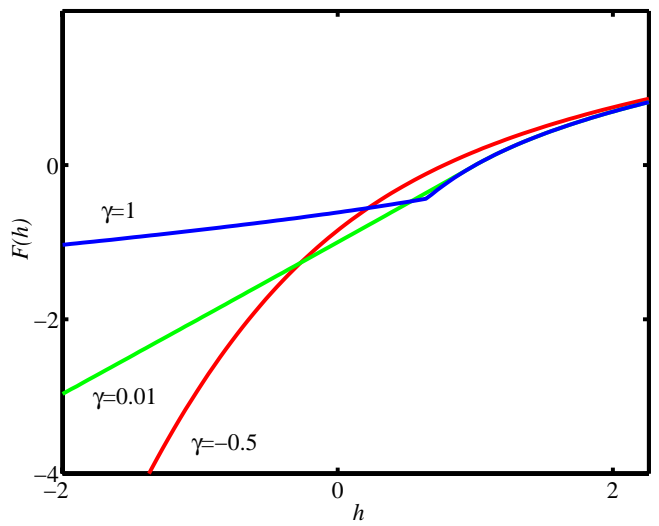

Figure 2: The static return function $F$ written as a function of $h=[(1-\delta) k-$ $\left.k^{\prime}\right] k^{-\alpha}$. Note the distinct kinks when $\gamma>0$ : here the planner switches from working hard to not working at all.

in our model. However, as we show in this section, the planner's labor supply function can also feature jumps up. Thus the limits of the cycle can themselves shift over time.

Intuitively, as the planner gets richer (begins with more capital), the wealth effect leads him to work less but the productivity effect leads him to work more. This latter effect emerges because labor's product is proportional to $k^{\alpha}$. In the neoclassical growth model capital provides two services: it is a productivity-enhancing input to production and it is a risk-free storage technology. When $\gamma<0$, there is normally no benefit to using capital for storage. When $\gamma>0$, though, the optimal production plan may feature periods of hard work interspersed with vacations. In the face of such a production plan, society must have a storage technology in order 
to smooth consumption over time. As we have seen, the only storage technology available is one that also augments labor productivity, giving rise to even more complicated dynamics than a cycle with constant limits.

With a finite horizon, there is some terminal period $T$, beyond which the household (and hence the social planner) does not survive. In this ultimate period, $t=T$, we know that the optimal investment policy satisfies $k_{T+1}=0$. As a result, we can write down the period- $T$ value function:

$$
V_{T}\left(k_{T}\right)=X\left(k_{T}, 0\right)=\alpha \log \left(k_{T}\right)+F\left(k_{T}, 0\right) .
$$

We know from lemma 2 above that if $\gamma>0, F\left(k_{T}, 0\right)$ will feature a kink at a critical capital level, $\tilde{k}_{T}>0$, where the optimal labor supply jumps down to zero.

Working backwards from the terminal period $T$, the planner's value function in the penultimate period, $T-1$, is:

$$
V_{T-1}\left(k_{T-1}\right)=\max _{k_{T}} X_{T-1}\left(k_{T-1}, k_{T}\right)+\beta X\left(k_{T}, 0\right) .
$$

This function has, potentially, two or more local maxima. The discontinuous jumps in the planner's policy functions will be associated with jumps from one local maximum to another.

Imagine fixing the penultimate period's capital stock, $k_{T-1}$, and varying terminal capital, $k_{T}$, beginning from zero and moving up. At very low choices of $k_{T}$, the planner will not work today (because investment is low), but will work tomorrow (because $k_{T}$ is low). Thus the penultimate period's static return func- 
tion, $X_{T-1}$, will have a relatively high (negative) slope in $k_{T}$ : because the planner is not working in period $T-1$, changes in $k_{T}$ affect consumption one-for-one. By contrast, the ultimate period's static return function, $X_{T}$, will have a relatively small (positive) slope in $k_{T}$ : because the planner is working in period $T$, changes in $k_{T}$ have a less than one-for-one effect on consumption.

At higher choices of $k_{T}$, though, the situation is reversed. Now the planner is working in period $T-1$ (because investment is high) and not working in period $T$ (because the planner has inherited a relatively high capital stock). Thus the relative magnitudes of the slopes of the static return functions, $X_{T-1}$ and $X_{T}$, will be reversed. Now $X_{T-1}$ slopes down less and $X_{T}$ slopes up more.

As a result, there will be (at least) two local solutions to the planner's problem; in one, he is not working today but is working tomorrow, in the other, he is working today but is not working tomorrow. In both cases there will be an optimal choice of investment. The planner's global problem is to choose which of these local solutions is best. One can also imagine other solutions to the planner's problem, associated with other policy combinations, e.g. working in both periods.

The planner's dilemma is displayed in figure 3 below, where we plot the planner's period $T-1$ value function as a function of initial capital (which the planner inherits) and terminal capital (which the planner must choose):

$$
V_{T-1}\left(k_{T-1} ; k_{T}\right)=X_{T-1}\left(k_{T-1}, k_{T}\right)+\beta X_{T}\left(k_{T}, 0\right) .
$$

We mark the global maxima over choices of terminal capital. Notice that the two 
local maxima occur at widely separated choices of terminal capital. As initial capital increases, the right hand maximum, associated with high labor today and low labor tomorrow, increases relative to the left hand maximum.

More generally, notice that the relative magnitudes of the local maxima will depend on the planner's penultimate wealth, $k_{T-1}$; however, the planner will be trading off the wealth effect and the productivity effect of capital. At low values of $k_{T-1}$, the marginal utility of consumption will be high, prompting the planner to work, but the marginal product of labor, which depends on $k_{T-1}^{\alpha}$, will be low, prompting the planner not to work. At high values of $k_{T-1}$ the opposite is true. We know from lemma 2 that under no circumstances will be the planner be willing to work a non-zero amount less than $\gamma / \phi$. Thus even if the planner could equate the marginal value of the consumption produced by labor with the (constant) marginal cost of labor at a low level of labor, the planner would spurn such a solution.

In the special case that $\alpha=0$ the productivity effect vanishes. The planner's policy functions will still have downward discontinuities, but the jumps up vanish. When $\alpha=0$ capital is only useful as a low-return storage technology In this case, the marginal product of labor will be constant no matter what the capital stock. Thus we can think about the planner's problem as one of minimizing the effort cost necessary to finance an optimal stream of consumption. We explore this special case in some detail below.

The planner's optimal choices of investment, $k_{T}\left(k_{T-1}\right)$ and effort $n_{T-1}, n_{T}$ as a function of the penultimate period's capital stock are displayed in figure 4 below. Notice that labor in period $T-1$ and investment both jump up at precisely 
the values of initial capital, $k_{T-1}$, where the global maximum switched from one local maximum to the other. Also notice that at this critical level of capital the planner's optimal choice of labor effort in the next period, period $T$, falls to zero, even as the planner's optimal choice of labor effort in the current period, period $T-1$, jumps up.

The policies displayed in figure 4 have other notable features. First, effort in period $T-1$ is increasing in the inherited capital stock, $k_{T-1}$. Here the productivity effect is dominating (notice that these figures use a large value for capital's social product, $\alpha=1$ ); at low levels of initial capital, labor's product is too low to bother working (consumption can be read as the difference between the 45 degree line and the optimal choice of $k_{T}$ ). Second, effort in period $T$ is decreasing in the initial capital stock, $k_{T-1}$. Because in the second period the capital stock will be completely consumed, the wealth effect dominates. Third, effort, $n_{T-1}$, and investment, $k_{T}$, display several (small) jumps at relatively high values of the initial capital stock, $k_{T-1}$. These are the result of the competing wealth and productivity effects. Notice that effort tends to gradually rise, the result of the productivity effect augmenting labor's product, and then suddely fall, the result of the wealth effect.

More generally, with multiple periods, the relevant objective functions can feature multiple local maxima and hence several jumps. 


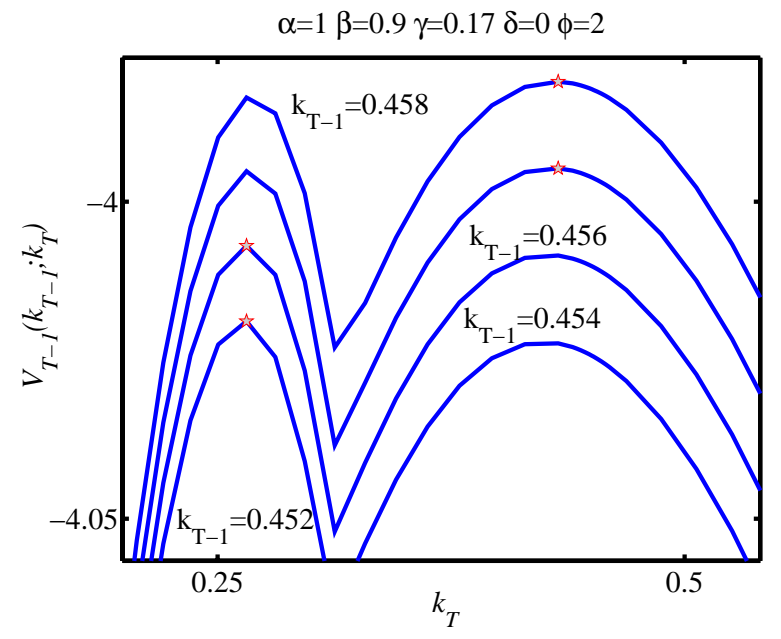

Figure 3: The planner's penultimate period value function, $V_{T-1}$, over choices of investment, $k_{T}$, for several different levels of initial capital, $k_{T-1}$. The stars give the global maxima.

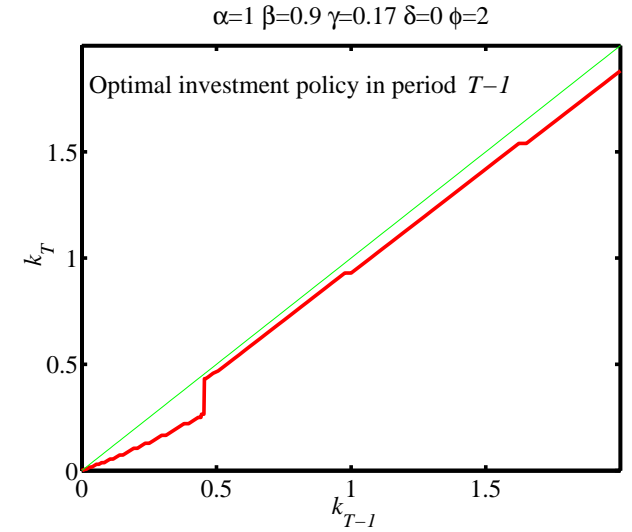

a. Investment

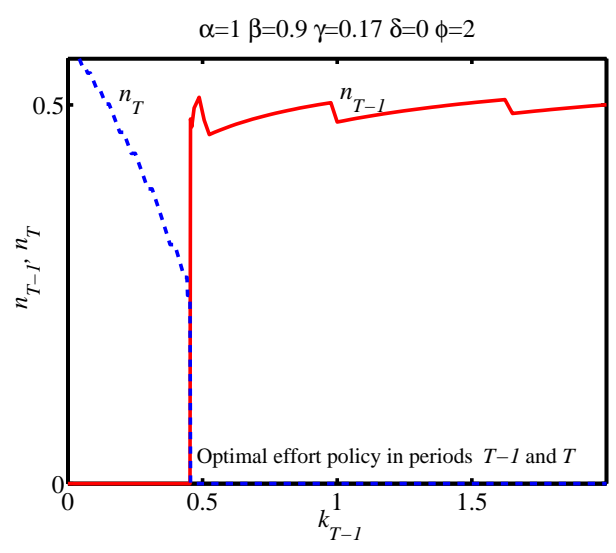

b. Labor

Figure 4: Optimal investment policies, $k_{T}\left(k_{T-1}\right)$, and labor policies, $n_{T-1}\left(k_{t-1}\right)$ and $n_{T}\left(k_{T}\left(k_{t-1}\right)\right)$ in the finite-horizon case. 


\subsection{Stochastic Control}

Until we now we have forced the planner to use policies that are non-stochastic; the planner simply chooses effort and investment levels given an initial capital level. The non-convexity in the period return function, $F$, identified in lemma 2, suggests that the planner would use lotteries if permitted. In this section we liberalize the planner's control set to permit stochastic policies. We show that, in certain cases, the planner would in fact use lotteries to increase expected utilities; however, these lotteries ultimately take the form of gambles over the current capital stock. In our setting, such lotteries would impose aggregate risk, and would require a counterparty willing to take the other side of a gamble involving a significant part of a national capital stock. As a result, such gambles are probably not a feature of the U.S. economy as whole. On the other hand, they may be feasible for particular sectors of the U.S. economy and for smaller, developing countries.

As the horizon faced by the planner grows to infinity, the region of capital stocks within which the planner would use lotteries may shrink to nothing. By sharply varying labor and investment over time, the planner may be able to get arbitrarily close to the value function with lotteries. We show that in the case where capital is purely a storage technology, so that $\alpha=0$, even at the infinitehorizon limit the planner benefits from using lotteries.

Lotteries over inputs to a production function have appeared in other applications. Prescott and Townsend (1984a,b) laid the theoretical framework for their use when contracts must satisfy an incentive compatibility constraint. Phelan and Townsend (1991) demonstrated how to compute multi-period and infinite-horizon 
planner's problems using lottery contracts. Lehnert (1998) used this framework to characterize the effect of stochastic capital inputs on growth models. Most recently, Paulson and Townsend (2000) have found some empirical evidence for the presence of lottery-based contracts in micro-level data from Thailand.

\section{Generalized Planner's Problem With Lotteries}

We follow Phelan and Townsend (1991) in generalizing our model to allow the planner to use lotteries. As before, we require capital and effort to live in the closed and bounded intervals of the real line, $\mathbf{K}=\left[k_{\min }, k_{\max }\right], k_{\min }>0$ and $\mathbf{N}=\left[0, n_{\max }\right]$. We now generalize the planner's choice set each period to be a probability measure over joint events $\mathbf{S}$ in $\mathfrak{B}(\mathbf{S})$, where $\mathbf{s}$ is a triplet $\left(n, k^{\prime}, k\right), \mathbf{S}$ is the cross-product $\mathbf{N} \times \mathbf{K} \times \mathbf{K}$ and $\mathfrak{B}(\mathbf{S})$ are all the Borel subsets of $\mathbf{S}$. The planner's problem now becomes:

$$
\begin{aligned}
& W(k)=\max _{\xi} \int_{\left(n, k^{\prime}, z\right) \in \mathbf{N} \times \mathbf{K} \times \mathbf{K}}\left\{\log \left(z^{\alpha} n^{1+\gamma}+(1-\delta) z-k^{\prime}\right)-\phi n\right. \\
& \left.+\beta W\left(k^{\prime}\right)\right\} \xi\left(n, k^{\prime}, z\right) d\left(n, k^{\prime}, z\right) \text {. }
\end{aligned}
$$

The choice object $\xi\left(n, k^{\prime}, k\right)$ is best thought of as the joint probability density over today's effort $n$, tomorrow's capital stock $k^{\prime}$ and today's capital stock $k$ (which we denoted with the dummy variable $z$ in the planner's problem, equation 3.9). 
This optimization proceeds subject to an economy-wide resource constraint:

$$
\int_{\left(n, k^{\prime}, z\right) \in \mathbf{N} \times \mathbf{K} \times \mathbf{K}} z \xi\left(n, k^{\prime}, z\right) d\left(n, k^{\prime}, z\right)=k .
$$

This constraint requires that the expected value of the capital input actually used in production be equal to the total outstanding stock of capital. Thus the planner can neither gain (nor, of course, lose) capital in expected value. An alternate view would be to rule out all lotteries that vary the capital input away from the inherited, beginning-of-period capital stock. Such a constraint would be:

$$
\xi\left(n, k^{\prime}, z\right)=0, \text { all } z \neq k .
$$

This constraint forces the planner to put measure zero on any outcome that varies today's capital input $z$ from its initial level $k$, but the planner may still use stochastic choices of investment and labor effort.

Both constraints (C1) and (C2) are linear in the choice object $\xi$ and define closed and convex sets of permitted choices $\xi$. We can therefore define two additional operators as lottery-based counterparts to the operator $\mathcal{T}$ defined in equation (3.5) above. Each new operator will be associated with a different restriction on lotteries. As before, the operators map $\mathbb{B}(\mathbf{K})$ into itself:

$$
\begin{aligned}
& \mathcal{T}_{\mathrm{c1}} f=\max _{\xi} \int_{\mathbf{S}}\left\{\log (c)-\phi n+\beta f\left(k^{\prime}\right)\right\} \xi(\mathbf{s}) d(\mathbf{s}), \text { subject to }(\mathrm{C} 1) \\
& \mathcal{T}_{\mathrm{c} 2} f=\max _{\xi} \int_{\mathbf{S}}\left\{\log (c)-\phi n+\beta f\left(k^{\prime}\right)\right\} \xi(\mathbf{s}) d(\mathbf{s}), \text { subject to }(\mathrm{C} 2)
\end{aligned}
$$


Here we have suppressed for notational convenience the relationships:

$$
\begin{aligned}
& c=k^{\alpha} n^{1+\gamma}+(1-\delta) k-k^{\prime}, \text { and: } \\
& \mathbf{s} \in \mathbf{S}=\mathbf{N} \times \mathbf{K} \times \mathbf{K} .
\end{aligned}
$$

From Phelan and Townsend (1991) we know that the operators $\mathcal{T}_{\mathrm{C} 1}$ and $\mathcal{T}_{\mathrm{C} 2}$ are

contraction mappings. Thus we define two additional value functions as the fixed points of the appropriate operators:

$$
W_{(C 1)}(k)=\left(\mathcal{T}_{\mathrm{Cl}} W_{(C 1)}\right)(k) \text { and } W_{(C 2)}(k)=\left(\mathcal{T}_{\mathrm{C} 2} W_{(C 2)}\right)(k)
$$

\section{Results Without Capital Gambles}

Define a capital gamble as any policy $\xi$ that satisfies constraint $(\mathrm{C} 1)$ but violates (C2). Such policies involve variations of the capital input away from the beginning-of-period inherited capital stock. As we discussed earlier, capital gambles are probably not feasible for large economies taken as a whole. Our first result shows the effect of ruling capital gambles out:

Lemma $3\left(\mathcal{T}_{C 2} f\right)(k)=(\mathcal{T} f)(k)$ all bounded and continuous functions $f$.

Notice as an immediate consequence of lemma 3 we know that the the fixed points of the two operators, $\mathcal{T}$ and $\mathcal{T}_{\mathrm{C} 2}$, must be equal, hence the value functions $W_{(C 2)}(k)$ and $V(k)$ must be equal. 


\section{Results With Capital Gambles}

We now explore the consequences for our model of allowing the planner to use fair gambles over the inherited capital stock. In addition to arbitrary bounded and continuous functions $f: \mathbf{K} \rightarrow \mathbf{R} \subset \mathbb{R}$, we will find it convenient to define the zero function as $f^{(0)}=0$ all $k \in \mathbf{K}$.

Lemma 4 The operator $\mathcal{T}_{C l}$ satisfies:

1. $\left(\mathcal{T}_{\text {Cl }} f\right)(k) \geq(\mathcal{T} f)(k)$ all $k \in \mathbf{K}$,

2. $\gamma<0 \Longrightarrow\left(\mathcal{T}_{\text {Cl }} f^{(0)}\right)(k)=\left(\mathcal{T} f^{(0)}\right)(k)$ all $k \in \mathbf{K}$,

3. $\gamma \geq 0 \Longrightarrow\left(\mathcal{T}_{\text {Cl }} f^{(0)}\right)(k)>\left(\mathcal{T} f^{(0)}\right)(k)$ at least one $k \in \mathbf{K}$.

Notice what lemma 4.3 does not say, namely, that an economy with gambles would be strictly better off than an economy without gambles at the infinitehorizon limit. Instead, the lemma applies only to the finite horizon case, when the continuation utility is given by the zero function $f^{(0)}$. Iterating backwards, however, the planner without capital gambles can choose policies that cleverly mimic lotteries, and, indeed, can often (but not always) achieve the same utility as in the world with capital gambles. That is, for certain parameters, it is the case that $V(k)=W_{(C 1)}(k)$. However, we can identify one functional form where this is not the case:

Theorem 3 If $\alpha=0$ then:

1. If $\gamma<0$ then $W_{(C 1)}(k)=V(k)$ all $k \in K$. 
2. If $\gamma>\gamma^{\star}$ where:

$$
\gamma \geq \gamma^{\star} \equiv \frac{\beta}{1+\beta} \frac{\log \left[((1-\delta) \beta)^{-1}\right]}{\log (1+\beta)}>0
$$

then $W_{(C 1)}(k) \geq V(k)$ all $k \in K$ and $W_{(C 1)}(\hat{k})>V(\hat{k})$ at least one $\hat{k} \in K$.

Intuitively, when $\alpha=0$, we saw in section 3.3 that the productivity effect vanishes and capital is only used as a low-return storage technology. Thus we can imagine breaking the planner's problem into two pieces. First the planner chooses a time path for consumption that does not depend on the capital stock and then decides how much to work in order to finance this consumption stream. When $\gamma$ is large enough to overcome the effects of capital's depreciation rate, $\delta$, and the discount factor, $\beta$, it is optimal for the planner to bunch production; moreover, the planner decides how hard to work (in those periods that he does work) by choosing the number of vacation periods that a particular level of effort will net him. ${ }^{4}$ Imagine beginning with initial capital of zero: the planner works hard enough to finance, say, a three-period vacation. If the planner were instead endowed with a small positive amount of capital, he responds by working a little less, but maintaining the same target level of capital next period (enough to finance a three-period vacation). As the stock of initial capital grows, though, the planner's choice of how much to invest can jump down. Here the planner switches to a policy of working hard enough to finance a two period vacation. Before the jump, the planner's value function will have a relatively shallow slope in initial

\footnotetext{
${ }^{4}$ Note that when capital does not depreciate, so $\delta=0$, and the discount factor approaches unity, $\gamma^{\star}$ approaches zero.
} 
capital; the planner is only adjusting one margin, how much to work. After the jump, the planner's value function will have a relatively steeper slope because the planner has adjusted the amount of target capital next period. A capital-gamble style lottery would allow the planner to convexify the kink, increasing the value function in expected value.

\section{Numerical Results}

Although we were able to make some progress in characterizing the planner's problem in discrete time, a complete analytic characterization is impossible without assuming constant returns to scale in capital, which we have seen rules out optimal cycles. In this section we characterize the solution to the planner's problem using numerical techniques. We begin by (briefly) justifying the technique we use, we then establish a benchmark specification of parameters and vary parameters of interest around the benchmark. Next, we explore the implications of theorem 3 by solving the planner's problem when $\alpha=0$ with and without lotteries. One final question of interest is whether or not the solution at a particular parameter combination will feature endogenous cycles; we explore this issue by solving the model at many combinations of parameters and finding the regions in parameter space where cycles arise. 


\subsection{Numerical Technique}

Because our basic problem is, from the standpoint of numerical techniques, quite straightforward, we can afford to use one of the simplest numerical techniques available, namely discrete-state dynamic programming. We specify a grid of capital points $\mathrm{K}$ :

$$
\mathrm{K}=\left\{k_{\min }, k_{2}, \ldots, k_{m-2}, k_{m-1}, k_{\max }\right\}, k_{\min }>0 .
$$

Here the integer $m$ is the number of grid points in the capital state space approximation. Associated with every combination of capital today $k$ and tomorrow $k^{\prime}$, where $k, k^{\prime}$ are in $\mathrm{K}$, we compute the static return $\mathrm{F}$, defined in equation (3.8) above:

$$
\mathrm{F}\left(k_{i}, k_{j}\right)=\max _{n}\left\{\log \left(k_{i}^{\alpha} n^{1+\gamma}+(1-\delta) k_{i}-k_{j}\right)-\phi n\right\}, \text { all } k_{i}, k_{j} \in \mathrm{K}
$$

(Notice that by working hard enough, the planner can realize positive consumption for any combination $k_{i}, k_{j}$, as long as $k_{i}>0$.) Associated with each $k_{i}, k_{j}$ there will be an optimal choice of labor effort $n_{i j}$. For values of $k_{i}, k_{j}$ such that:

$$
h_{i j} \equiv \frac{(1-\delta) k_{i}-k_{j}}{k_{i}^{\alpha}}>h_{\max }
$$

we know that the optimal solution cannot be to work hard, so that $n_{i j}=0$. If $h_{i j} \leq h_{\max }$, we solve the planner's first order condition (given in equation A.2) to find a candidate solution involving high labor effort. If $h_{i j}>0$, we also have 
to check the corner, $n_{i j}=0$, because the planner might prefer not working to working. With the static return function F computed, we next solve a recursive version of the planner's problem:

$$
\mathrm{v}^{(\ell+1)}\left(k_{i}\right)=\max _{k_{j}}\left\{\mathrm{~F}\left(k_{i}, k_{j}\right)+\beta \mathrm{V}^{(\ell)}\left(k_{j}\right)\right\}, \text { where } k_{j} \in \mathrm{K} ; \text { all } k_{i} \in \mathrm{K} .
$$

We begin with an initial guess at the value function $\mathrm{v}^{(0)}$ and implement the discrete operator defined by equation (4.1) until successive iterations produce value functions that are numerically indistinguishable from one another. See the discussion in Judd (1999) for a complete discussion of this technique.

\subsection{Benchmark Parameterization}

Because the case that we are interested in (when $\alpha<1$ and $\gamma$ is relatively low) is precisely the case without an analytic solution, we pick a central benchmark calibration for our parameters and then vary parameters to study their effect on our model. The benchmarks and variation ranges are given in table 1 below.

The parameter of primary interest in our model is $\gamma$, which governs the total returns to labor, $1+\gamma$. We first vary this parameter, then the returns to capital $\alpha$ (when $\alpha>0$ ), then the depreciation rate $\delta$ and finally the discount factor $\beta$.

Our chosen benchmark is $\gamma=1 / 6, \alpha=1 / 3, \beta=0.98, \delta=0.1$ and $\phi=$ 2. We specify a relatively low value of returns to labor, $1+\gamma$, to demonstrate that optimal labor cycles can occur under mild increasing returns to labor. The return to capital parameter, $\alpha$, is chosen to match the social returns to capital 
used in many business cycle models. The total returns to scale in the benchmark specification equal 1.5. Next, we select a discount factor of $\beta=0.98$ and a depreciation rate of $\delta=0.1$, which are reasonable calibrations for an annual model. In the sensitivity analysis, we show that labor cycles appear under a larger discount factor or a smaller depreciation rate.

\section{Varying Returns to Labor: $\gamma$}

Figure 5 plots the optimal investment (that is, next period's capital) and labor policy functions for the benchmark parameterization and two alternate levels of $\gamma$. Figure 6 shows the dynamics of labor effort, capital and consumption under the benchmark and alternate levels of $\gamma$. As expected, the pronounced, discontinuous drop in the policy functions, when they occur along the optimal capital accumulation path, generate cycles. (Interestingly, although consumption cycles can be eliminated, the planner chooses not to.) Notice also that the limits of the cycles shift over time; this is the result of the discrete jumps up in the policy functions, discussed in section 3.3 above.

The labor and capital policy functions for all three parameterizations feature downward discontinuities, where the social planner chooses to stop working and consume only out of the capital stock. Examining the labor policy functions, a larger $\gamma$ implies a higher level of the labor input for any level of current capital. Likewise, for the three values of $\gamma$ considered here, a larger $\gamma$ is associated with a higher cut-off capital stock for supplying labor. As predicted by lemma 2.3 above, the minimum positive labor value (the smallest non-zero amount of labor 


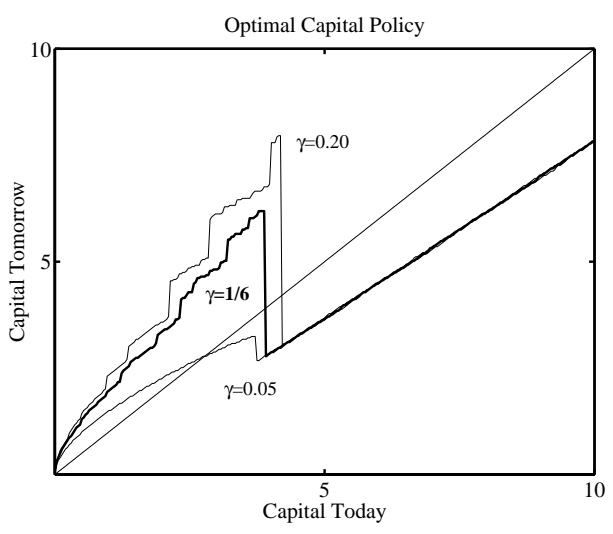

a. Investment

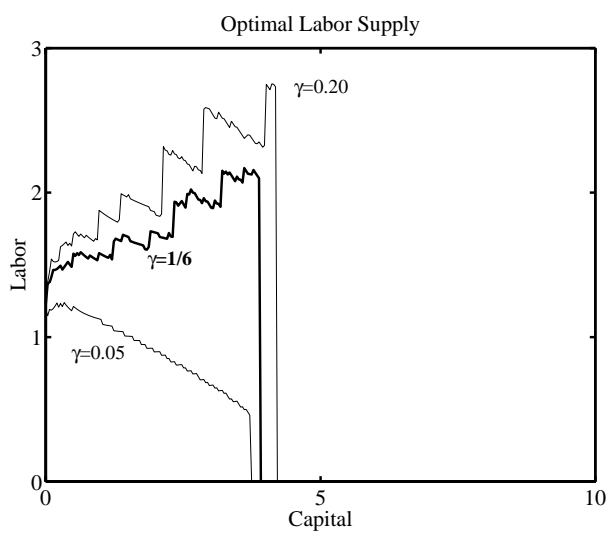

b. Labor

Figure 5: Optimal investment and labor policies (as a function of capital) for varying levels of $\gamma$.

that the planner is willing to supply) also increases in $\gamma$. Since the returns to labor are higher, the optimal allocation involves greater labor effort for any level of the capital stock. Examining the capital policy function, a larger $\gamma$ implies a higher level of (next period) capital for any current capital stock.

In the cases of $\gamma=1 / 6$ or 0.2 , the optimal capital policy function does not cross the 45 degree line at a continuous point; instead, at a point-call it $\tilde{k}$-the optimal investment policy features a discontinuous jump down. For both of these parameterizations, the optimal capital sequence does not converge asymptotically, but instead cycles between periods of positive labor input and savings to periods of zero labor input and dissaving. Intuively, the social planner would like to find an intermediate average level of the capital stock at which to spend his time saving, consuming and working. Such an ideal capital stock may not exist under increasing returns. Low capital stocks do not exploit capital's ability to augment 

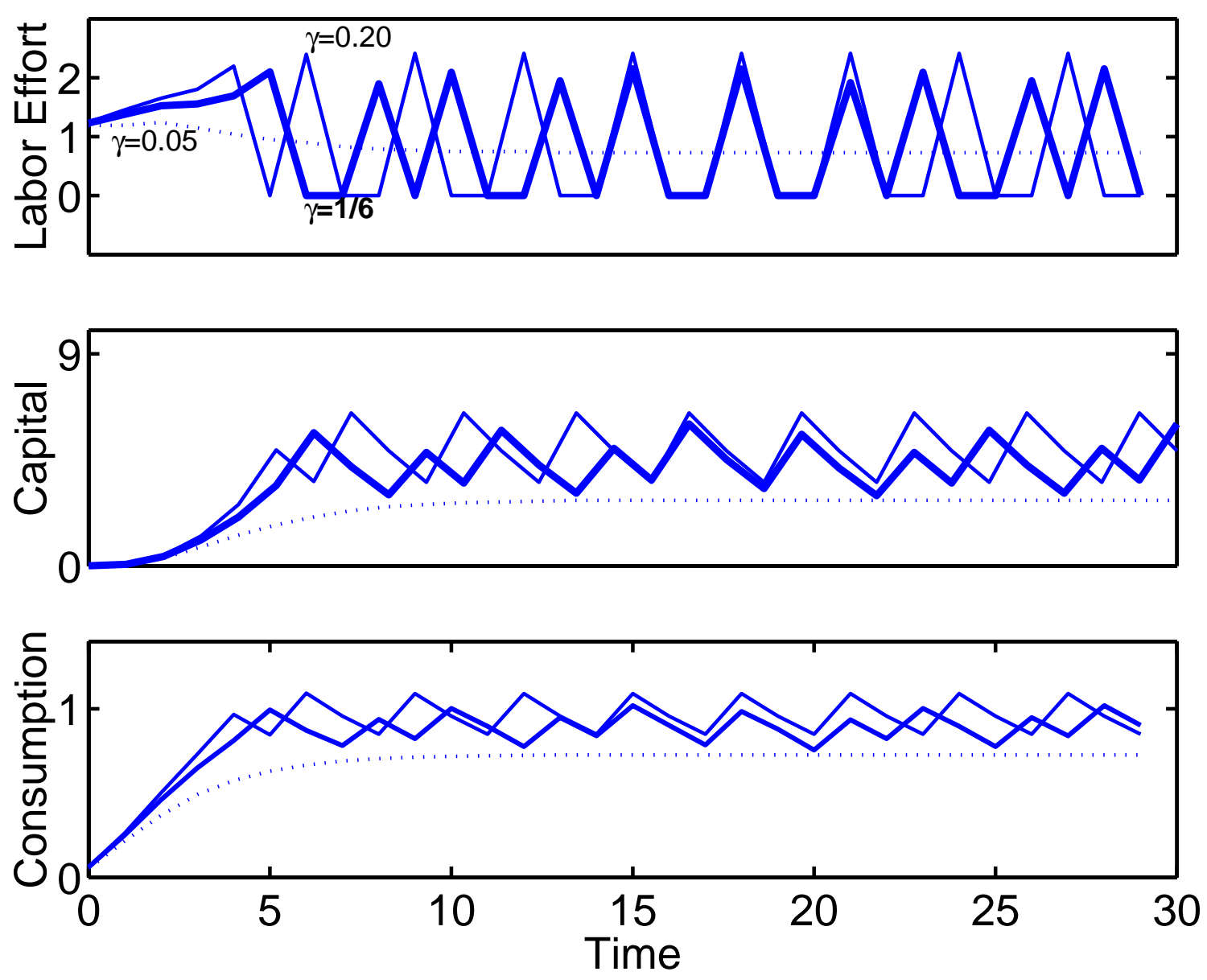

Figure 6: Dynamic paths of labor effort, capital stock and consumption under different values of $\gamma$. 
labor productivity, while high capital stocks are expensive to maintain because of capital's diminishing marginal product. By oscillating labor supply, the social planner takes advantage of increasing returns to labor by jumping between positive (and high) labor and zero labor. Notice than in our example, the planner works hard in bursts of one period only; however, the planner's vacations are typically longer than a single period. In order to smooth consumption and keep capital near its target level, when the planner is following an oscillating labor supply policy, capital must be above the steady-state when labor is zero because households consume out of the capital stock. Positive consumption and capital depreciation imply capital falls during zero labor periods. On the other hand, capital is below the steady-state when labor is positive. To finance consumption in the future zero labor phase of the cycle, the social planner increases the capital stock during the positive labor phase of the cycle.

For both the $\gamma=1 / 6$ and $\gamma=0.2$ cases, the capital and labor policy functions display several upward discontinuities when $k<\tilde{k}$. There are the discontinuities discussed in section 3.3 above.

An oscillating labor plan has one built-in inefficiency: the period of high labor effort occurs when the capital stock is lowest, but a low capital stock implies a relatively low marginal product of labor. If the degree of increasing returns is sufficiently low, oscillating labor supply will not be optimal. This occurs when $\gamma=0.05$ : although the policy functions continue to display the characteristic downward discontinuity, it occurs at capital levels above the steady-state. The capital policy function in this case crosses the 45 degree line continuously and 
from above, so there exists a steady-state optimal capital stock. Labor cycling is not optimal because the benefit of bunching production in the face of small increasing returns is outweighed by the cost of having to work when the capital stock is low.

\section{Varying the Depreciation Rate: $\delta$}

In our model, capital provides two services to households: (i) it is a risk-free constant returns storage technology, with a rate of return decreasing in $\delta$, and (ii) an investment which augments the productivity of next period labor. We can vary (i) by changing the depreciation rate.

Figure 7 plot the optimal investment and labor policy functions for three different depreciation rates $\delta=0.1$, the benchmark case, $\delta=0.075$. and $\delta=0.25$. There is a general downward shift in both policy functions as $\delta$ rises. As capital depreciates more quickly, the planner reduces gross physical investment and labor. Also, the point at which labor input goes to zero as a function of the current capital stock decreases as $\delta$ rises. For the largest depreciation rate, $\delta=0.25$, the optimal allocation converges to a constant labor and capital steady-state.

\section{Varying the Returns to Capital: $\alpha>0$}

One parameter of particular interest is the return to capital, $\alpha$. Theorem 3 characterizes the planner's problem in the special case that $\alpha=0$; further, in section 4.3 below we compute numerical solutions using lotteries in that case. However, it is also interesting to study the effect of increasing $\alpha$ outside of this special case. 


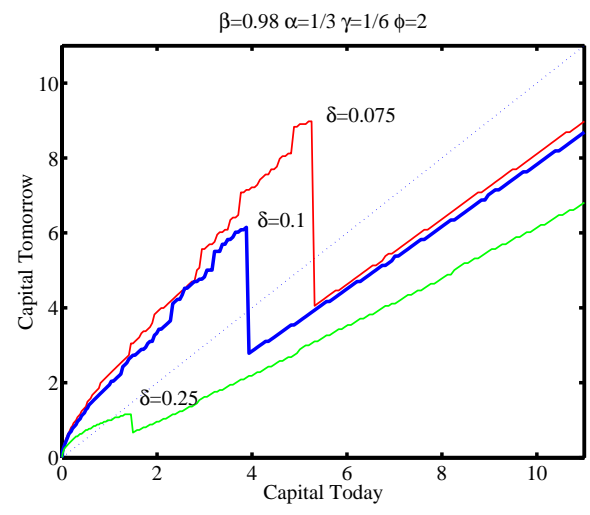

a. Investment policy

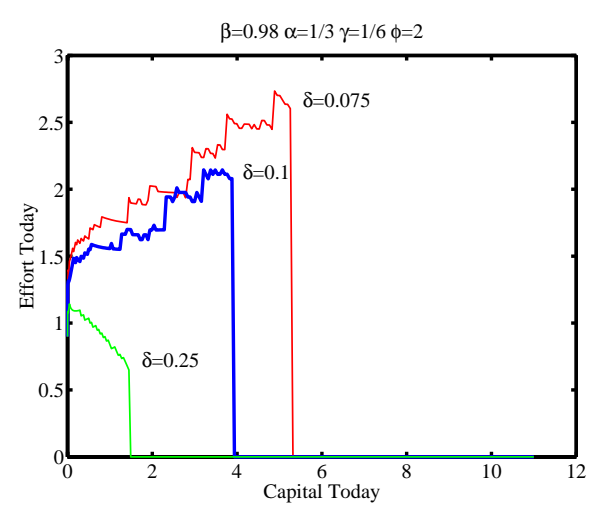

b. Labor policy

Figure 7: Effect of varying the depreciation rate, $\delta$.

Figure 8 displays the optimal policies for three levels of $\alpha,\{1 / 6,1 / 3,1 / 2\}$.

As $\alpha$ increases, the critical capital level at which the investment policy jumps down, $\tilde{k}$, shifts out. In all cases (as, indeed, in the case when $\alpha=0$ ) endogenous cycles arise. Thus the average capital level during oscillations is increasing in $\alpha$. Finally, notice that the sharp jumps up in the policy functions appear at all levels of $\alpha$, so the limits of the cycles will also shift.

\section{Varying the Discount Factor: $\beta$}

The discount factor $\beta$ defines the length of the period. As $\beta$ approaches 1 , the discrete time model becomes more like the continuous model. Low values of $\beta$ correspond to a longer horizon during which the planner must fix inputs.

We consider three levels for $\beta,\{0.8,0.98,0.99\}$. In figure 9 , we plot the investment and labor policy functions for the benchmark model at these three discount factors. As $\beta$ rises, the policy functions all shift up, implying higher average 


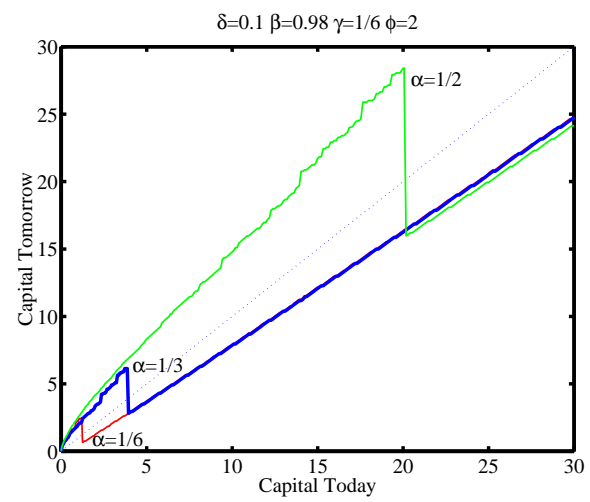

a. Investment policy

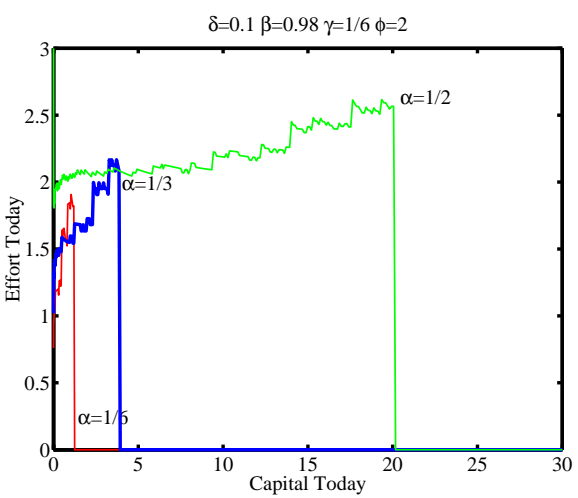

b. Labor policy

Figure 8: Effect of varying returns to capital, $\alpha$.

capital and effort levels. Also, the critical capital level $\tilde{k}$ shifts to the right.

For the lowest value of $\beta, 0.8$, cycles do not arise endogenously. This arises from the discrete-time formulation; from our analysis of the benchmark model, the optimal allocation involves keeping capital close to a target steady-state. Such a policy becomes more difficult to implement using cycles if the planner is forced to maintain a labor choice over a longer and longer horizon.

\subsection{Capital Gambles When $\alpha=0$}

Theorem 3 assures us that capital gambles will strictly improve welfare (in expected value) when $\alpha=0$ and $\gamma$ is large enough to stimulate cycles. Under our benchmark parameterization, the critical value of $\gamma$ is about 0.0910 ; thus our central level of $\gamma, 1 / 6$, is large enough to provoke cycles and hence for lotteries to be welfare-improving. We now display some numerical results for our benchmark model (with $\alpha=0$ ) with and without capital gambles. 


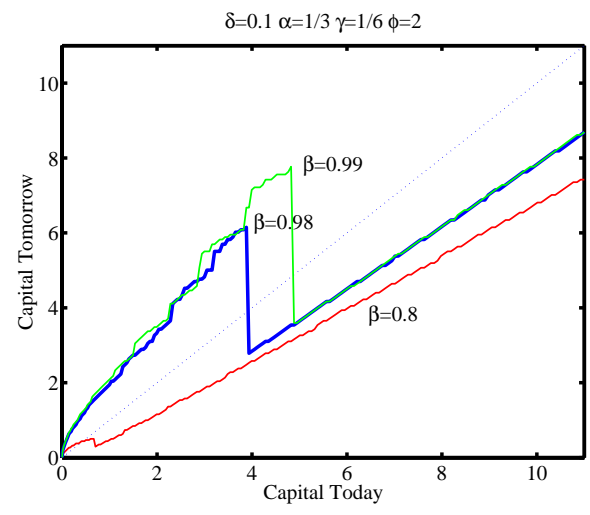

a. Investment policy

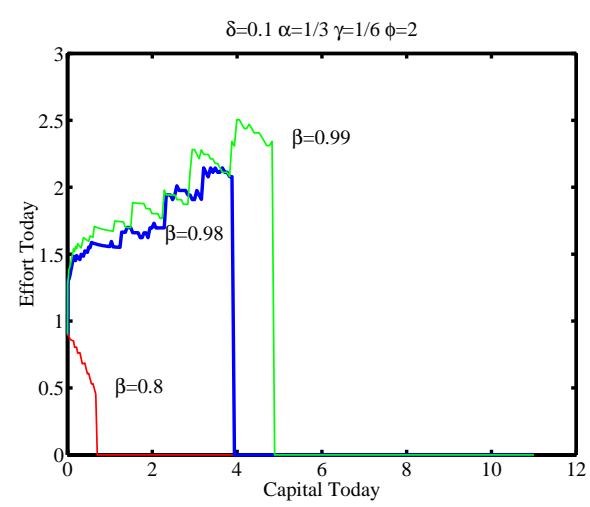

b. Labor policy

Figure 9: Effect of varying the discount factor, $\beta$.

We calculated the optimal stochastic policy using the linear programming techniques pioneered by Phelan and Townsend (1991). Our particular application here of these techniques is quite straightforward; unlike Phelan and Townsend's problem, our model does not feature moral hazard or exogenous shocks, so our lotteries do not have to satisfy incentive-compatibility or Bayes-consistency constraints. In the same way that we compute non-stochastic policies using discretespace approximations to the operator $\mathcal{T}$, we compute optimal stochastic policies using a discrete-space approximation to the operator $\mathcal{T}_{\mathrm{C} 1}$. Instead of choosing a general measure over the Borel subsets of effort, investment and capital, we choose a probability mass function over investment and capital grid points.

Figure 10 displays the levels and slopes of $W_{(C 1)}(k)$ and $V(k)$, the value functions with and without capital-gamble style lotteries. Without lotteries, the value function $V$ displays several distinct kinks, while the planner uses lotteries to convexify around these kinks, so that $W$ is linear in these regions. The effect of 
lotteries on the slopes of the value functions is quite striking: without lotteries, the slope of the value function swings wildly, while with lotteries it is smoothly decreasing, and flat at the kinks in $V$. Notice that the planner does not always use lotteries; in regions where $V$ is not kinked, the planner can do no better with lotteries than without them.

Figure 11 displays the associated investment and effort policies. Notice that, in the case of lotteries, we display the expected values of investment and labor. The planner is using lotteries to bounce between two extreme points-high labor and high investment against low labor and low investment. As a result, even though the expected value of next period's capital level crosses the 45-degree line smoothly and from above, there is no steady-state capital in the usual sense. Instead, the planner is undertaking ex ante capital gambles between a higher and a lower capital stock.

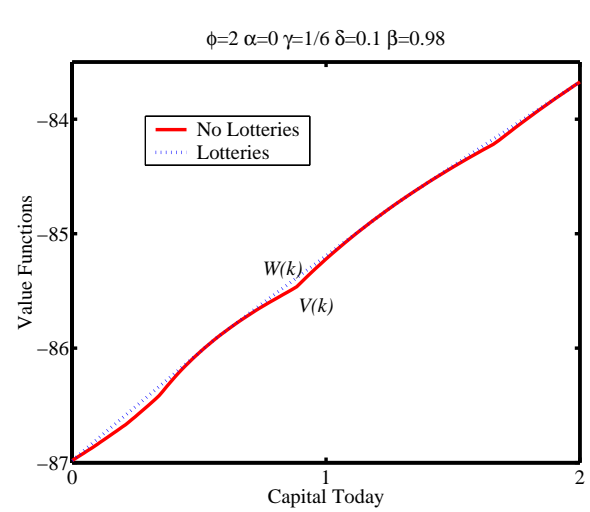

a. Levels

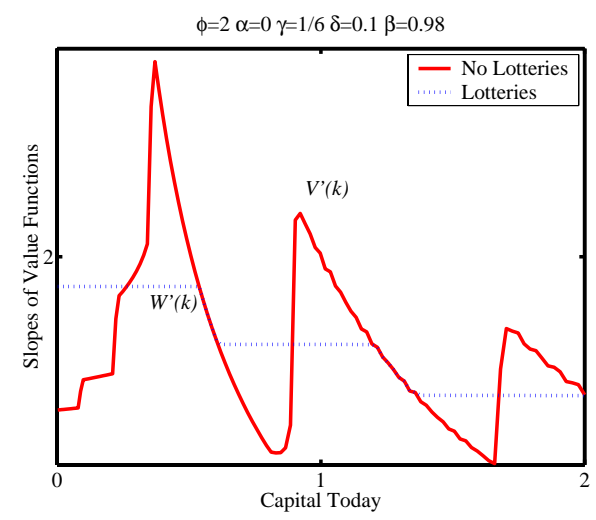

b. Slopes

Figure 10: Value functions with and without capital gambles. 


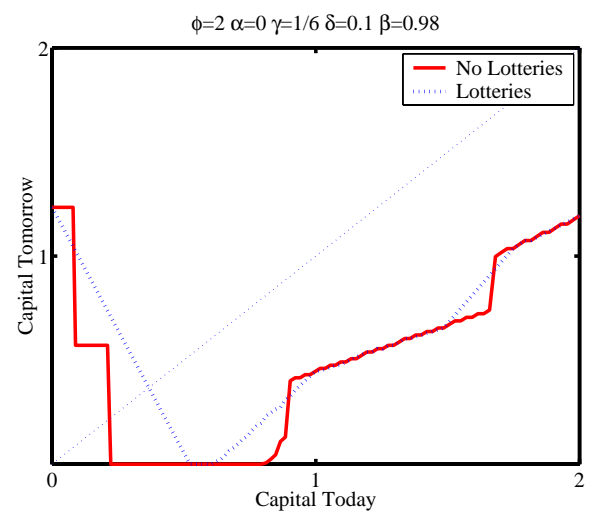

a. Investment

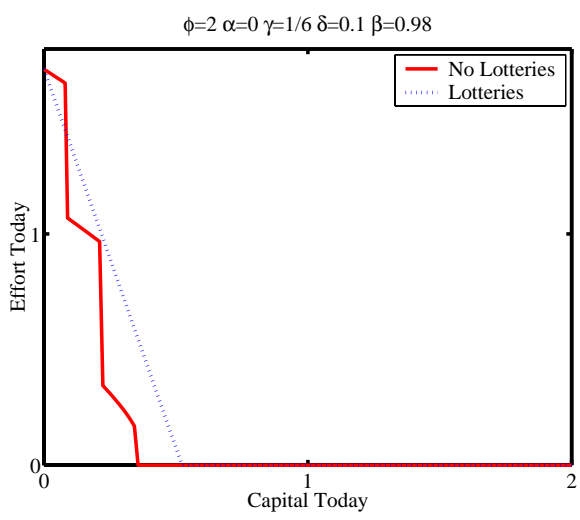

b. Labor

Figure 11: Optimal policies with and without capital gambles.

\subsection{Optimality of Cycles}

We now present results from jointly varying the parameters $\alpha, \beta, \gamma$ over a wide range. Instead of presenting the policy functions in each case, we determine whether, under each parameter combination, the policies give rise to endogenous cycles. Figure 12 displays the results in $(\alpha, \gamma)$ space, and figure 13 displays the results in $(\beta, \gamma)$ space. The results show that cycles are less likely at lower values of $\beta$; a higher value of $\gamma$ is required to overcome the natural tendency of the planner to converge to a fixed capital stock as the horizon grows. Also, as $\alpha$ grows, cycles become less likely. Thus as the model approaches Christiano and Harrison's $\alpha=1$ case, cycles gradually vanish from the model. 
Table 1: Benchmark parameterization and variations

\begin{tabular}{llccc}
\hline \hline & Parameter & $\min$ & benchmark & $\max$ \\
\hline$\gamma$ & Labor's externality & $-1 / 3$ & $1 / 6$ & 2 \\
$\alpha$ & Capital's product & 0 & $1 / 3$ & 0.8 \\
$\beta$ & Discount factor & 0 & 0.98 & 0.999 \\
$\delta$ & Capital survival & & 0.10 & \\
$\phi$ & Disutility of labor & 2 & \\
\hline \hline
\end{tabular}

Optimal Cycles: $\beta=0.98 \delta=0.1 \phi=2$

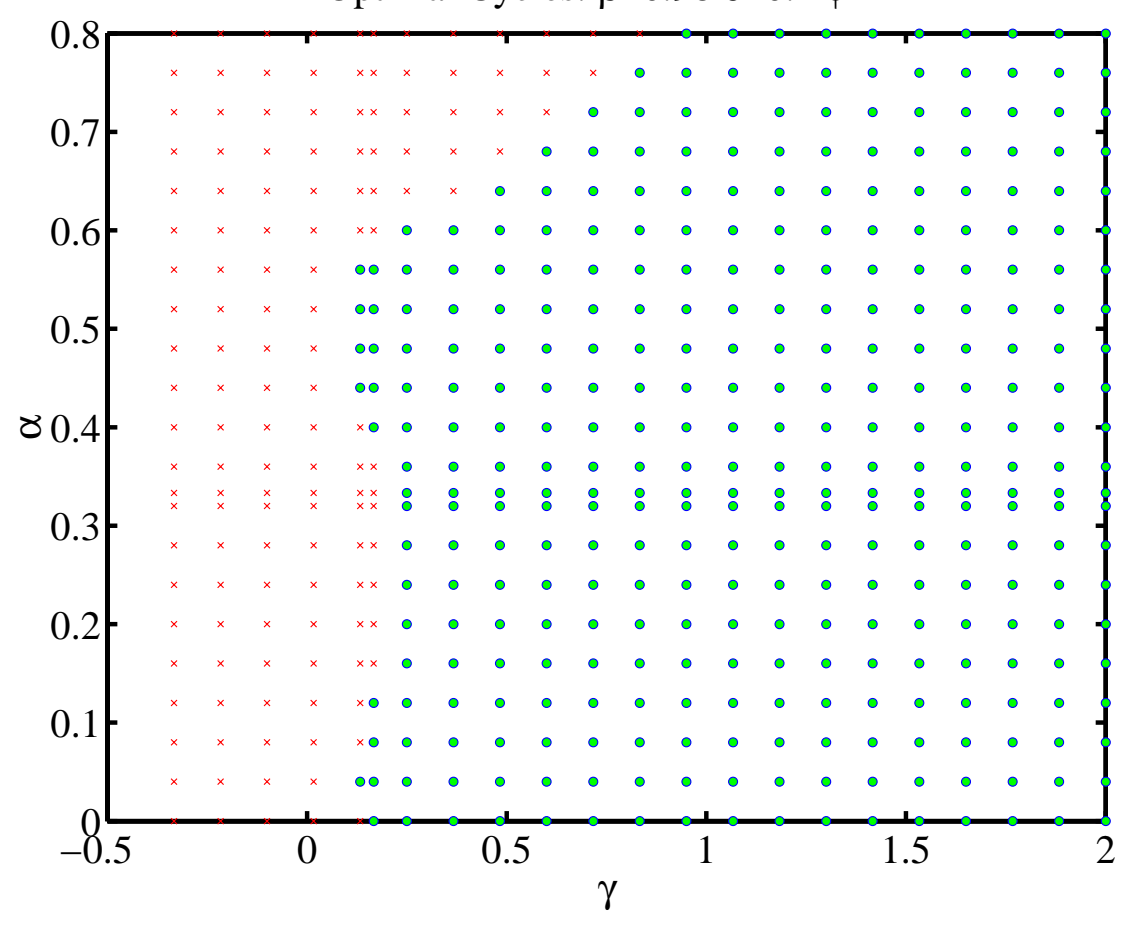

Figure 12: Cycles in $(\alpha, \gamma)$ space. 


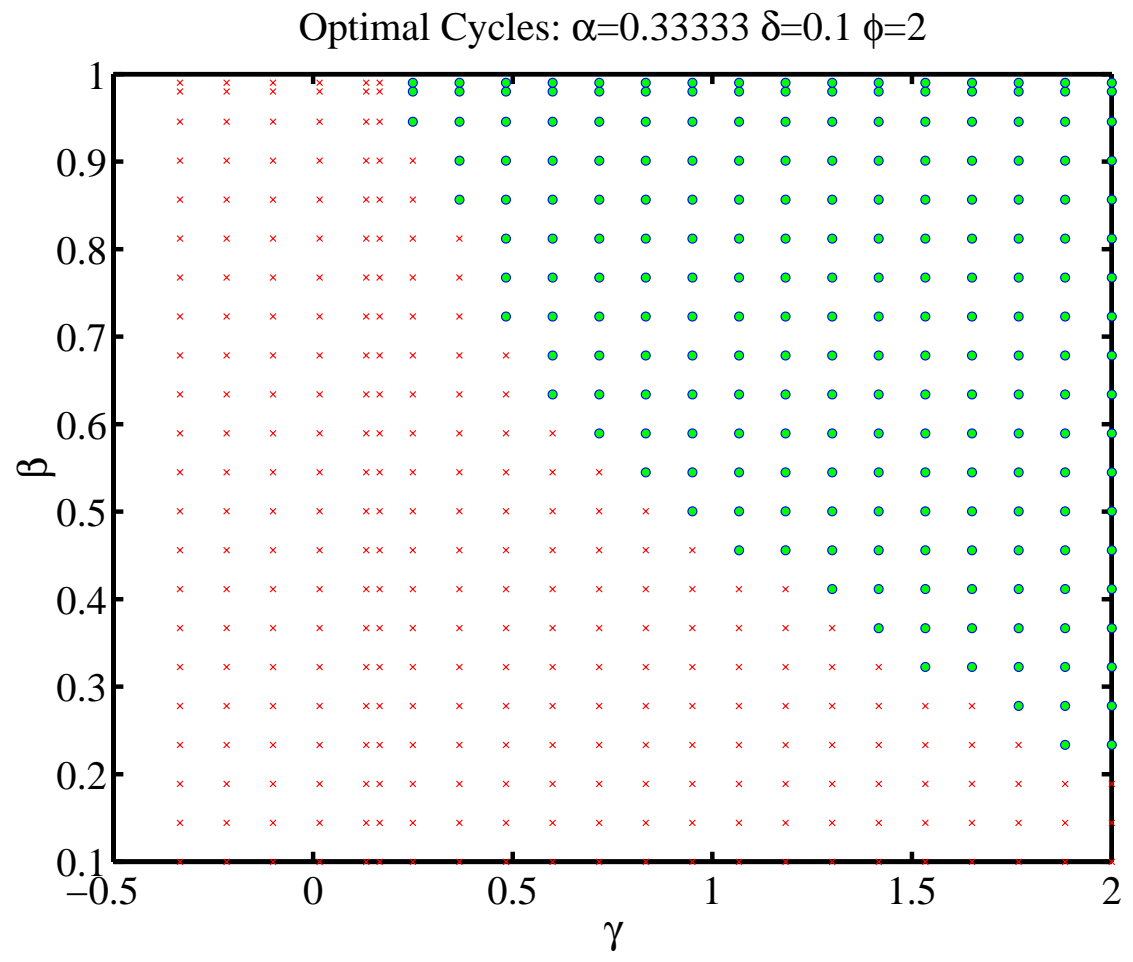

Figure 13: Cycles in $(\beta, \gamma)$ space. 


\section{Conclusion}

Consider two economies, called "A" and "B". In both economies technological progress allows workers to communicate their ideas, needs and resources much more effectively than before; so much so, in fact, that labor productivity noticeably increases even as the aggregate supply of labor also increases. In economy A, thanks to strenuous interventions by the government, investment, employment and output fluctuate little. In economy B, by contrast, investment, employment and output fluctuate, often apparently at random. In economy B, inventories of goods accumulate towards the ends of booms, and then run down, signaling the beginning of recession. Employment peaks, paradoxically, exactly when the capital stock has run down significantly and thus augments labor's productivity the least. In which economy are households better off?

This paper is, at least in part, a plea not to dismiss the possibility that economy $\mathrm{B}$ is actually following the optimal path. We showed that, within an increasingreturns model, multiple competitive equilibria exist precisely in those cases when it is at least possible that the optimal path of output is not constant.

Indeed, we showed that the optimal policy under increasing returns can feature (1) endogenous cycles, (2) shifting endpoints for these cycles and (3) stochastic capital gambles. None of these phenomena are normally taken as the hallmarks of wise economic policy; and yet, in certain cases in our model, they are precisely that.

Our results abstracted from many features of the real world, and thus should 
not be taken as a positive prescription to allow (or even encourage) cycles. In particular, the sole welfare cost of our cycles came from the fluctuations in aggregate consumption. In reality, because households retain a significant amount of idiosyncratic risk, it is likely that some fraction of households would bear most of the consumption declines associated with the cycles. 


\section{A Proofs}

\section{A.1 Proof of Theorem 1}

We can easily compute the eigenvalues $\nu$ of the linear system given by equation (2.15) by solving the characteristic equation:

$$
\operatorname{det}(A-\nu I)=0 \text {. }
$$

The eigenvalues $\nu$ are the solution to the quadratic equation:

$$
\nu^{2}-\operatorname{tr}(A) \nu+\operatorname{det}(A)=0 .
$$

Applying the quadratic formula:

$$
\nu=\frac{1}{2}\left[\operatorname{tr}(A) \pm \sqrt{(\operatorname{tr}(A))^{2}-4 \operatorname{det}(A)}\right] .
$$

The eigenvalues will have opposite signs if and only if $\operatorname{det}(A)<0$. Thus, for any given starting value of capital, there will a unique path to the steady-state if and only if $\operatorname{det}(A)<0$. In the same way, for any given starting value of capital close to the steady-state, there will be many paths to the steady-state (that is, both values of $\nu$ will be negative) if and only $\operatorname{det}(A)>0$ and $\operatorname{tr}(A)<0$.

The determinant is given by:

$$
\operatorname{det}(A)=\frac{(1-\alpha)(\rho+\delta)}{\gamma}\left[\frac{\rho+\delta}{a}-\delta\right] .
$$

Given our previous assumptions on the parameters, $0<a<\alpha<1$ and $\rho, \delta>0$, it must be true that:

$$
\operatorname{sign}(\operatorname{det}(A))=\operatorname{sign}(\gamma)
$$

Notice that $\gamma>0$ is sufficient to rule out the usual local saddle-path stability. Condition 2 of the theorem gives the negative trace condition.

\section{A.2 Proof of Theorem 2}

Proof by contradiction. Assume first that there exist paths for capital, consumption and labor $k^{\star}(t), c^{\star}(t), n^{\star}(t)$ where the control function is piecewise contin- 
uous and the state is piecewise differentiable, that maximizes the social welfare function, equation (2.18). According to the maximum principle, at each instant the current value Hamiltonian is maximized at $c^{\star}(t), n^{\star}(t)$. Then:

$$
\begin{aligned}
& \lambda_{0}[\log (c)-\phi n]+\lambda\left[\left(k^{\star}\right)^{\alpha} n^{1+\gamma}-c-\delta k^{\star}\right] \\
& \quad \leq \lambda_{0}\left[\log \left(c^{\star}\right)-\phi n^{\star}\right]+\lambda\left[\left(k^{\star}\right)^{\alpha}\left(n^{\star}\right)^{1+\gamma}-c^{\star}-\delta k^{\star}\right] \text { for all }(c, n) \in U
\end{aligned}
$$

For the particular problem that we are studying, households can never be satiated; thus the objective can never be disregarded. Thus we can dispense with the case of $\lambda_{0}=0$, where the Hamiltonian would be strictly decreasing in consumption, and increasing in labor effort.

We wish to show that an alternate choice of policies, $\left(c^{\star}, \theta n^{\star}\right)$, increases the value of $H^{c}$ above $\left(c^{\star}, n^{\star}\right)$ for some $\theta>0$. Plugging in our conjectured improved control pair, the above inequality becomes:

$$
\lambda\left(k^{\star}\right)^{\alpha}\left(n^{\star}\right)^{1+\gamma}\left(\theta^{1+\gamma}-1\right) \leq \phi(\theta-1) n^{\star} .
$$

Let $v(\theta)$ and $w(\theta)$ denote the left and right-hand side of (A.1). The functions coincide when $\theta=1$, and both are differentiable in $\theta$. If $v^{\prime} \neq w^{\prime}$ at $\theta=1$, then the functions cross at this point, and condition (A.1) must be violated for some $\theta$. The more interesting case, when $v^{\prime}=w^{\prime}$ is illustrated in figure 14 below. If $\gamma>0$, then $v$ is convex and increasing in $\theta$ and $v>w$ for $\theta \neq 1$. 


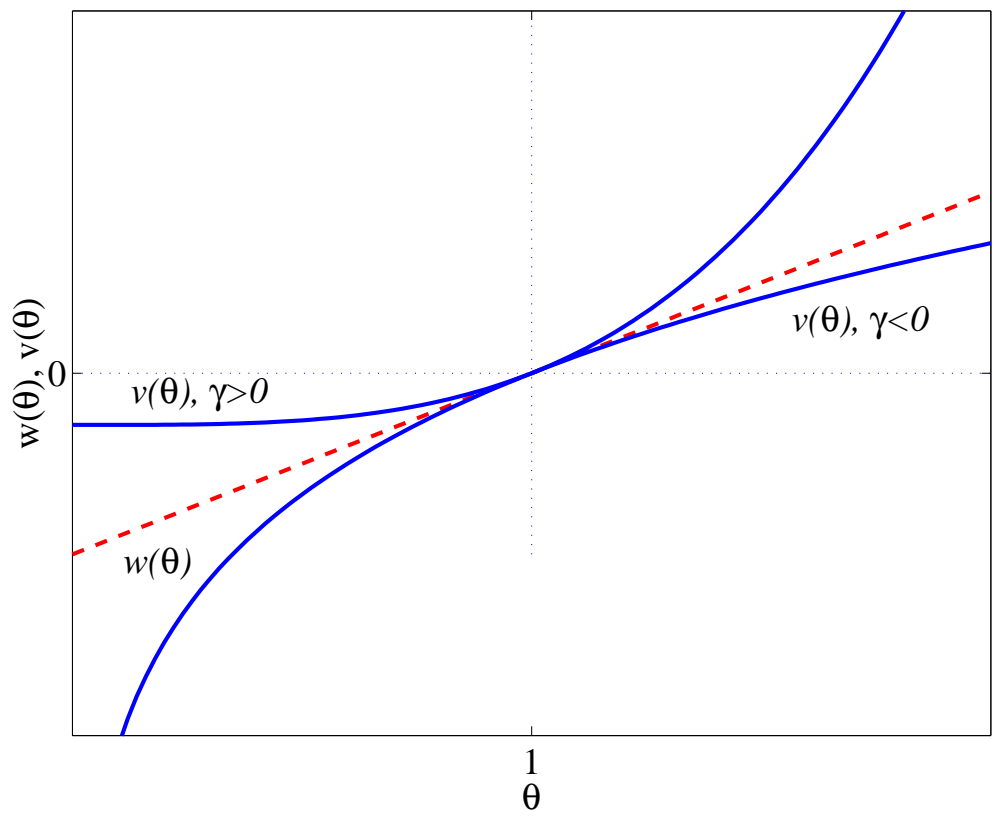

Figure 14: Relation of $v$ and $w$. 


\section{A.3 Proof of Lemma 1}

We first show that $n^{\star}\left(k, k^{\prime}\right)$ is bounded, and then it a natural result that $F$ is also bounded.

First notice that the variable $h=\left[(1-\delta) k-k^{\prime}\right] k^{-\alpha}$ defined in equation (3.7) above is sufficient for $F\left(k, k^{\prime}\right)$, so that $F$ is a function of $k$ and $k^{\prime}$ only as they affect $h$. Now we can write the derivative of $F$ as:

$$
F_{n}\left(h\left(k, k^{\prime}\right) ; n\right)=\frac{(1+\gamma) n^{\gamma}}{n^{1+\gamma}+h}-\phi
$$

Thus if we were to imagine the planner choosing an unboundedly large level of effort, we can find the limit of this derivative:

$$
\lim _{n \rightarrow \infty} F_{n}(h ; n)=-\phi<0 .
$$

If $\gamma>0$, the function $F$ is non-monotone in $n$ and also non-concave, thus opening the possibility of a corner solution. However, if $h>0$ and $\gamma>0$, then $F_{n}(h ; n=$ $0)=-\phi$ also. The planner might like to force labor effort even lower than zero, but, of course, cannot. Thus it is the left constraint we have to check.

So now we have established that $F$ is the upper envelope of two functions: $\bar{F}$ and $\underline{F}$. These functions are defined as:

$$
\begin{aligned}
\bar{F}\left(h\left(k, k^{\prime}\right)\right) & =\log \left(\bar{n}^{1+\gamma}+h\right)-\phi \bar{n}, \text { where: } \\
\bar{n} & : \bar{n}^{1+\gamma}-[(1+\gamma) / \phi] \bar{n}^{\gamma}+h=0, \text { and } \\
\underline{F}\left(h\left(k, k^{\prime}\right)\right) & =\log (h) .
\end{aligned}
$$

If there is no interior solution to the planner's first order condition (A.4), we take it as undefined. Figure 15 below plots $\bar{F}, \underline{F}$ and their upper envelope.

We are guaranteed that $h$ is finite by our assumptions on $\mathbf{K}$. This then in turn guarantees us that $\bar{n}$ (if it exists) is also finite. Optimal labor $n^{\star}$ is either 0 or $\bar{n}$, both of which are finite. But if $n$ and $h$ are finite, $F$ must also be bounded.

\section{A.4 Proof of Lemma 2}

To show lemma 2.1, we show that $F\left(k, k^{\prime} ; n\right)$ is strictly concave in $n$ if $\gamma<0$, so that the planner is always picking an interior choice of $n, n>0$. The second 


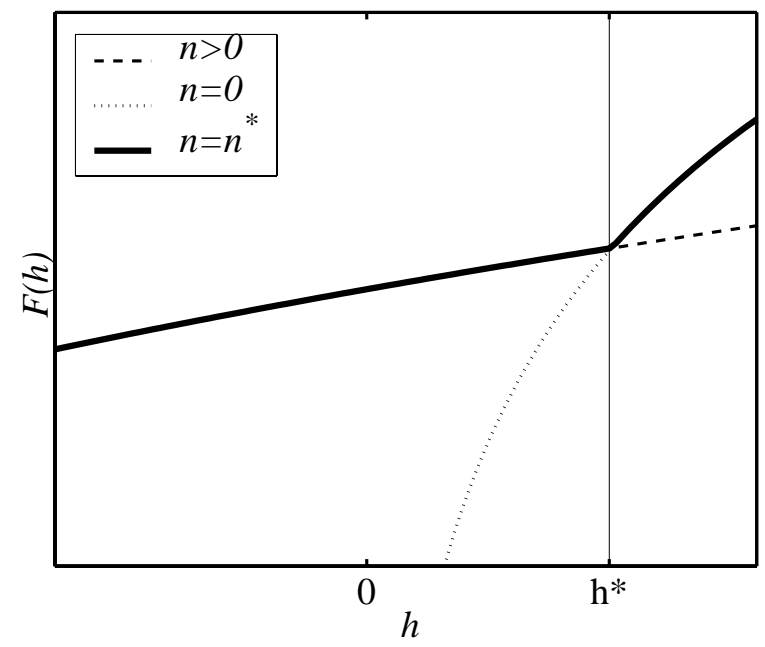

Figure 15: Static return $F$.

derivative of $F$ is given by:

$$
\begin{aligned}
\frac{d^{2}}{d n^{2}} F\left(k, k^{\prime} ; n\right) & =\frac{\gamma}{n} M(n)-[M(n)]^{2}, \text { where: } \\
M(n) & \equiv \frac{(1+\gamma) n^{\gamma}}{n^{1+\gamma}+h} .
\end{aligned}
$$

Here $h$ is the convenient function of $k$ and $k^{\prime}, h=\left[(1-\delta) k-k^{\prime}\right] k^{-\alpha}$ first defined in equation (3.7) above. When $\gamma<0, M(n)>0$ if $n>0$, so the sign of the second derivative of $F$ in $n$ is certainly negative if $\gamma$ is negative.

To show lemma 2.2, notice first that an interior solution to the static problem requires that labor be chosen so that $F_{n}\left(h\left(k, k^{\prime}\right) ; \bar{n}\right)=0$, or $H(n)+h=0$, where:

$$
H(n) \equiv n^{1+\gamma}-\frac{1+\gamma}{\phi} n^{\gamma}
$$

Positive values of $h$ are associated with negative investment and increased consumption today relative to tomorrow. The larger $h$ is, the better off the planner is today. We show that $H(n)+h=0$ will always have a unique solution in $n$ if $\gamma<0$ but may have no solutions if $\gamma>0$ and $h$ is large and positive.

Notice that if $\gamma<0, H(n)$ becomes unboundedly negative as $n$ approaches 0 from the left; further, $H^{\prime}(n)$ is positive everywhere $n \geq 0$ so $H(n)$ is monotone 
increasing. Thus for all $h \in(-\infty, \infty)$ a single value of $n$ will satisfy the planner's first order condition in $n: H(n)+h=0$.

If instead $\gamma>0$, we show $H(n)$ has a finite minimum and hence for large positive values of $h$, no value of $n \geq 0$ satisfies $H(n)+h=0$. For such values of $h$ the planner must set effort to zero (although he may choose to do so at smaller values as well). The first and second derivatives of $H(n)$ are:

$$
\begin{aligned}
H^{\prime}(n) & =(1+\gamma) n^{\gamma-1}[n-(\gamma / \phi)] \\
H^{\prime \prime}(n) & =\gamma(1+\gamma) n^{\gamma-2}[n-(\gamma-1) / \phi] .
\end{aligned}
$$

Thus $H(n)$ forms an upward-pointing parabola in $n$ when $\gamma>0$, with a global minimum at $n=\gamma / \phi$. If there are two solutions to $H(n)+h=0$ we need only check the one to the right of the parabolic minimum, because the planner's objective function slopes down to the left of this point. At the parabolic minimum, $H$ takes on the value:

$$
H(\gamma / \phi)=-\frac{1}{\gamma}\left(\frac{\gamma}{\phi}\right)^{1+\gamma}
$$

Note that $H(\gamma / \phi)<0$ in the $\gamma>0$ case. Thus for values of $h$ greater than $h_{\max }$ :

$$
h_{\max }=-H(\gamma / \phi)
$$

the planner's first order condition $H(n)+h=0$ has no non-imaginary solution, and the planner must set $n=0$.

The final element of the lemma, lemma 3, requires us to show that if $\gamma>0$, the planner's choice of optimal effort jumps down to zero at a certain point. In other words, there is some region of labor effort greater than zero which the planner never chooses. But we have seen that the planner never chooses labor effort $n<$ $\gamma / \phi$ as this puts him on the down-sloping part of the parabola $H(n)$.

\section{A.5 Proof that the Planner's Problem is a Contraction Map- ping}

The planner's problem is inherently a sequence problem. (Here we follow Stokey and Lucas (1989).) In equation (3.1) above we defined the planner's continuation 
utility at period $t$ when beginning with a capital stock of $k_{t}$ as $v_{t}\left(k_{t}\right)$. This is the solution to the sequence problem. In equation (3.4) above we wrote the planner's problem recursively and defined $V(k)$ at the value function. We now show that:

$$
v_{t}(k)=V(k), \text { all } k \in \mathbf{K} \text {. }
$$

We begin by re-writing the sequence problem using the static return function $X$ defined in equation (3.6) above:

$$
\text { (A.7) } v_{t}\left(k_{t}\right)=\max _{\left\{k_{t+i}\right\}_{i=0}^{i=\infty}} \sum_{i=0}^{\infty} \beta^{i} X\left(k_{t+i}, k_{t+i+1}\right), k_{t+i} \in \mathbf{K}, i=0,1,2, \ldots \infty \text {. }
$$

Next, we show that $v_{t}\left(k_{t}\right)$ must be finite for all starting values of capital. Define the sequence $\kappa_{t}$ as the optimal plan from the planner's sequence problem, (A.7) above:

$$
\kappa_{t}=\left\{k_{t+1}^{\star}, k_{t+2}^{\star}, \ldots\right\} .
$$

We now show that the present-discounted value of following the capital plan $\kappa_{t}$ from period $t$ forward is less than the present discounted value of an impossible plan. We then show that the PDV of this impossible plan is bounded. The particular impossible plan that we will consider will be this: the planner will follow the optimal path $\kappa_{t}$ going forward, except that each period we will allow him to completely consume the current capital stock without running it down; the next period, even after his capital binge, the capital stock called for by $\kappa_{t}$ appears (by magic). Thus the value of the optimal plan is given by:

$$
\begin{aligned}
\sum_{i=0}^{\infty} \beta^{i} X\left(k_{t+i}^{\star}, k_{t+i+1}^{\star}\right) & <\sum_{i=0}^{\infty} \beta^{i} X\left(k_{t+i}^{\star}, 0\right), \\
& \leq \sum_{i=0}^{\infty} \beta^{i} X\left(k_{\max }, 0\right),
\end{aligned}
$$


for simplicity, assume that $k_{\max }>h_{\max }$, defined in equation (A.6) above:

$$
\begin{aligned}
& =\sum_{i=0}^{\infty} \beta^{i} \log \left(k_{\max }\right) \\
& =\frac{1}{1-\beta} \log \left(k_{\max }\right)<\infty
\end{aligned}
$$

Next we turn to the recursive problem, equation (3.4) above. Define $\mathbb{B}(\mathbf{K})$ as a space of bounded continuous functions $f: \mathbf{K} \rightarrow \mathbf{R}$ (where $\mathbf{R} \subset \mathbb{R}$ ). Consider the functional operator $\mathcal{T}: \mathbb{B}(\mathbf{K}) \rightarrow \mathbb{B}(\mathbf{K})$ defined as:

$$
(\mathcal{T} f)(k)=\max _{k^{\prime} \in \mathbf{K}}\left\{X\left(k, k^{\prime}\right)+\beta f\left(k^{\prime}\right)\right\}, \text { all } k \text { in } \mathbf{K} .
$$

We now show that $\mathcal{T}$ satisfies Blackwell's sufficient conditions for a contraction, monotonicity and discounting.

First we show that $\mathcal{T}$ satisfies monotonicity. If the functions $f^{a}, f^{b}$ are both in $\mathbb{B}(\mathbf{K})$ and $f^{a}(k) \leq f^{b}(k)$ all $k$ in $\mathbf{K}$, we must show that $\mathcal{T} f^{a}(k) \leq \mathcal{T} f^{b}(k)$ all $k$ in $\mathrm{K}$ :

$$
\begin{aligned}
\left(\mathcal{T} f^{b}\right)(k) & =\max _{k^{\prime} \in \mathbf{K}}\left\{X\left(k, k^{\prime}\right)+\beta f^{b}\left(k^{\prime}\right)\right\} \\
& =\max _{k^{\prime} \in \mathbf{K}}\left\{X\left(k, k^{\prime}\right)+\beta f^{a}\left(k^{\prime}\right)+\beta\left[f^{b}\left(k^{\prime}\right)-f^{a}\left(k^{\prime}\right)\right]\right\} \\
& \geq\left(\mathcal{T} f^{a}\right)(k)+\beta \max _{k^{\prime}}\left\{f^{b}\left(k^{\prime}\right)-f^{a}\left(k^{\prime}\right)\right\} \\
& \geq\left(\mathcal{T} f^{a}\right)(k) .
\end{aligned}
$$

Next we show that $\mathcal{T}$ satisfies discounting. For any $f \in \mathbb{B}(\mathbf{K})$ we must show that there is $0<\eta<1$ such that $[\mathcal{T}(f+a)](k) \leq(\mathcal{T} f)(k)+\eta a$ for $a \geq 0$ and 
$k$ in $\mathbf{K}$ :

$$
\begin{aligned}
{[\mathcal{T}(f+a)](k) } & =\max _{k^{\prime} \in \mathbf{K}}\left\{X\left(k, k^{\prime}\right)+\beta f\left(k^{\prime}\right)+\beta a\right\} \\
& =\max _{k^{\prime} \in \mathbf{K}}\left\{X\left(k, k^{\prime}\right)+\beta f\left(k^{\prime}\right)\right\}+\beta a, \\
& =(\mathcal{T} f)(k)+\beta a .
\end{aligned}
$$

Therefore, any value for $\eta$ such that $\beta \leq \eta<1$ will satisfy the condition. We assumed that the discount factor $\beta$ was strictly between 0 and 1 .

\section{A.6 Proof of Lemma 3}

Begin by defining the budget set $\mathbf{B}(k)$ as:

$$
\mathbf{B}(k)=\left\{\left(n, k^{\prime}\right) \in \mathbf{N} \times \mathbf{K}: k^{\alpha} n^{1+\gamma}+(1-\delta) k-k^{\prime} \geq 0 .\right\}
$$

Notice parenthetically that if $\gamma>0, \mathbf{B}(k)$ is non-convex. Define $g_{k}$ to be the shared objective, $g: b \in \mathbf{B}(k) \rightarrow \mathbb{R}$ :

$$
g_{k}=\log \left(k^{\alpha} n^{1+\gamma}+(1-\delta) k-k^{\prime}\right)-\phi n+\beta f\left(k^{\prime}\right) .
$$

For all $k \in \mathbf{K}, g_{k}$ will be a finite, real-valued function of all points $b$ in the budget set $\mathbf{B}(k)$ except for those points at the edges of the budget set, where consumption is zero. At these points, $g_{k}$ will be negative infinity. Define the set of points $\mathbf{b}_{k}^{\star}$ as:

$$
\mathbf{b}_{k}^{\star} \equiv\left\{b \in \mathbf{B}(k): g_{k}(b) \geq g_{k}\left(b^{\prime}\right), \text { all } b^{\prime} \in \mathbf{B}(k) .\right\}
$$

Define $g_{k}^{\star}$ as $g_{k}(b)$ for $b$ in $\mathbf{b}_{k}^{\star}$. Notice that $g_{k}^{\star}$ will be finite, because for all $k>0$ the planner can find a policy $n, k^{\prime}$ that delivers non-zero consumption. Now, notice that from constraint $(\mathrm{C} 2)$ above, the choice object $\xi\left(n, k^{\prime}, z\right)$ must satisfy:

$$
\int_{\mathbf{N} \times \mathbf{K}} \xi\left(n, k^{\prime}, z\right) d\left(n, k^{\prime}\right)= \begin{cases}1 & z=k \\ 0 & z \neq k .\end{cases}
$$


We can therefore write the problem as one of choosing lotteries $\xi_{k}(b)$ for each capital stock $k$ and point $b$ in the budget set $\mathbf{B}(k)$. Therefore:

$$
\int_{\mathbf{B}(k)} g_{k}(b) \xi_{k}(b) d b \leq g_{k}^{\star} \text { all } \xi_{k}(b) .
$$

The best that the planner can do is write lotteries whose support is only $\mathbf{b}_{k}^{\star}$. But these lotteries can never produce outcomes greater than $g_{k}^{\star}$, which is available to the planner without lotteries. As a result, it must be the case that:

$$
\left(\mathcal{T}_{\mathrm{C} 2} f\right)(k)=(\mathcal{T} f)(k), \text { all } k \in \mathbf{K}
$$

This concludes the proof.

\section{A.7 Proof of Lemma 4}

The first item in the lemma is easy: because the planner can always replicate any non-stochastic plan with degenerate lotteries, the planner can never do worse with lotteries; thus $\mathcal{T}_{\mathrm{Cl}} f \geq \mathcal{T} f$.

Before addressing parts 2 and 3 of the lemma, we analyze the joint distribution $\xi$ further. Note that $\xi$ may be written as:

$$
\xi\left(n, k^{\prime}, k\right)=\pi(k) \zeta\left(n, k^{\prime} \mid k\right) .
$$

Here $\pi(k)$ is the capital gamble and $\zeta$ is the ex post lottery over effort and investment conditional on outcomes of the capital gamble. We know from lemma 3 that the conditional distributions $\zeta\left(n, k^{\prime} \mid k\right)$ are degenerate; thus the general contracts can be written as a stochastic part (the capital gamble) and a non-stochastic part (the optimal choice of effort and investment given an outcome $k$ from the capital gamble). Now the operator $\mathcal{T}_{\mathrm{C} 1}$ can be written as:

$$
\mathcal{T}_{\mathrm{C} 1} f=\max _{\pi} \int_{\mathbf{K}} \pi(z)\left\{\max _{n, k^{\prime}} \log \left(z^{\alpha} n^{1+\gamma}+(1-\delta) z-k^{\prime}\right)-\phi n+\beta f\left(k^{\prime}\right)\right\} d z .
$$

The constraint $(\mathrm{C} 1)$ is now written as:

$$
\int_{\mathbf{K}} \pi(z) z d z=k
$$

Turn now to item 2 of the lemma. Intuitively, without increasing returns to 
labor, the planner varies effort continuously with the capital stock. Because agents are risk-averse, the planner finds no benefit in using stochastic controls (or, of course, sharply varying effort and investment over time). If $\gamma<0$ then the static return function $F$ defined in equation (3.8) above is concave in $n$ for all $n \geq 0$. The planner's problem is now:

$$
\mathcal{T}_{\mathrm{C} 1}=\max _{\pi(k)} \int_{\mathbf{K}} \pi(k)\left\{\max _{n, k^{\prime}} \alpha \log (k)+F\left(k, k^{\prime} ; n\right)+\beta f^{(0)}\left(k^{\prime}\right)\right\} d k .
$$

The optimal investment policy is clearly degenerate, $k^{\prime}=0$ for all values of $k$. Further, because $F$ is concave for all $n \geq 0$, the optimal effort policy can be found at the critical point where $F_{n}\left(k, k^{\prime} ; \bar{n}\right)=0$, defined in equation A.2 above. Here:

$$
(\bar{n}(k))^{1+\gamma}-\frac{1+\gamma}{\phi}(\bar{n}(k))^{\gamma}+(1-\delta) k^{1-\alpha}=0,
$$

for all $k \in \mathbf{K}$. As a result, we know that if $\gamma<0$, optimal effort $n$ is non-zero and smoothly decreasing in $k$ for all $k>0$. Define $F^{(0)}(k)$ to be the static return function $F(k, 0 ; n)$ when effort $n$ is replaced by its optimal level, $\bar{n}$. The slope of $F^{(0)}(k)$ becomes:

$$
\frac{d}{d k} F^{(0)}(k)=\frac{d}{d k} F(k, 0 ; \bar{n})+\frac{d}{d n} F(k, 0 ; \bar{n}) .
$$

When $\gamma<0$ all of these derivatives exist and, from equation (A.8) above:

$$
\frac{d}{d n} F(k, 0 ; \bar{n})=0
$$

all $k>0$. Thus the second derivative is:

$$
\frac{d}{d k} F^{(0)}(k)=-\alpha(1-\alpha) \frac{k^{\alpha-2} n^{1+\gamma}+1-\delta}{c}-\frac{\left[k^{\alpha-2} n^{1+\gamma}+1-\delta\right]^{2}}{c^{2}}<0 .
$$

Thus the payoff function is strictly concave everywhere in $k>0$ and the planner has no motive to use lotteries. This gives us the second item in the lemma.

Turning now to the final item: when $\gamma \geq 0$, we show that the planner's optimal choice of labor effort will, for values of $k \geq \tilde{k}$, put the planner at the corner solution $n=0$, inducing a kink in the payoff function $F^{(0)}(k)$. Although the household is risk-averse, the sudden change in the slope of the payoff function 
will create a region where the household prefers capital gambles. To see this, consider again the locus of effort points defined by $F_{n}(k, 0 ; n)=0$, equation (A.8) above. This may be re-written as:

$$
n^{1+\gamma}-\frac{1+\gamma}{\phi} n^{\gamma}=-(1-\delta) k^{1-\alpha}
$$

For $\gamma \geq 0$, the left hand side (LHS) of this equation is convex increasing in $n \geq 0$. The global minimum is at the critical point $n=\gamma / \phi$, at which point the LHS is:

$$
\operatorname{LHS}\left(n=\frac{\gamma}{\phi}\right)=-\frac{1}{\gamma}\left(\frac{\gamma}{\phi}\right)^{1+\gamma}<0 .
$$

Thus for values of initial capital greater than the critical point $k_{\mathrm{CRIT}}$ :

$$
k_{\mathrm{CRIT}}=\frac{1}{1-\delta} \frac{1}{\gamma}\left(\frac{\gamma}{\phi}\right)^{1+\gamma},
$$

there is no real solution to equation A.8; for values of the capital stock $k>k_{\mathrm{CRIT}}$, the optimal policy must be to set effort to zero. At the other extreme, for very small values of initial capital $k \rightarrow 0$, we know that the optimal policy is to work hard. At some intermediate capital point, $0<\tilde{k} \leq k_{\mathrm{CRIT}}$, the policy switches from working hard to not working at all. We know that the slope of the value function $F^{(0)}(k)$ is lower at any capital level when the household works than when it does not (because it is consuming more in the former case than in the latter). Although the derivative of the value function is not defined at the kink $\tilde{k}$, we know that in an $\epsilon$-neighborhood of $\tilde{k}$ :

$$
\left.\lim _{\epsilon \rightarrow 0} \frac{d}{d k} F^{(0)}(k)\right|_{k=\tilde{k}-\epsilon}-\left.\frac{d}{d k} F^{(0)}(k)\right|_{k=\tilde{k}+\epsilon}>0 .
$$

Marginal utility is briefly increasing in wealth around the kink. In the neighborhood of $\tilde{k}$, the agent becomes in effect a risk-lover, prompting the social planner to implement capital gambles.

\section{A.8 Proof of Theorem 3}

We begin with part 1 of the theorem. We know from lemma 2 that when $\gamma<0$ the planner's static problem is smooth; his choice of labor effort never drops to zero 
no matter how rich he is. As a result, the planner's static return function $F$ does not feature a kink. But the planner's static objective is $X=\alpha \log (k)+F$; with the assumption that $\alpha=0$ this collapses to $X=F$, so we are also assured that the planner's static objective, $X$, is smooth and concave everywhere. From the proof of lemma 4 above we see that in such cases, there are no gains even in the finite horizon to capital gambles when the static objective is smooth. With a smooth and concave static objective, the infinite horizon value function inherits the properties of the static objective, and so is also smooth and concave. Thus there are no kinks to be convexified around with lotteries, and so capital gambles provide no service, and are ruled out by risk aversion.

The proof of the second part of the theorem proceeds as follows: (1) We establish that $\gamma \geq \gamma^{\star}$ is sufficient to establish that cycles are optimal; (2) We show that if cycles are optimal the planner works hard at low capital stocks but does not work at all at higher capital stocks; (3) We show that in the neighborhood of this critical capital region the planner's value function $V(k)$ is kinked; finally (4) we appeal to lemma 4 so that $\mathcal{T}_{\mathrm{C} 1} V(k)>V(k)$ in this neighborhood.

The planner's sequence problem is:

$$
\max _{\left\{c_{t}, n_{t}: t \geq 0\right\}} \sum_{t=0}^{\infty} \beta^{t}\left\{\log \left(c_{t}\right)-\phi n_{t}\right\},
$$

subject to: $k_{t+1}=(1-\delta) k_{t}+n_{t}^{1+\gamma}-c_{t}$, and $k_{t} \geq 0, t=1,2, \ldots$

If the non-negativity constraint on capital does not bind, the Euler equation implies that consumption follows the sequence:

$$
c_{t+1}=(1-\delta) \beta c_{t}
$$

But the non-negativity constraint will in general bind. If it binds every period, then the planner follows a constant-effort policy in which $k_{t}=0$ always. If it binds in some periods but not others, the planner follows a cyclical policy in which $k_{t}>0$ in some period but $k_{t}=0$ in others. Define $\bar{v}$ as the value of the constant-work policy:

$$
\bar{v}=\max _{c} \sum_{t=0}^{\infty} \beta^{t}\left\{\log (c)-\phi c^{1 /(1+\gamma)}\right\} .
$$

Now define a $T$-period cycle as a policy of working hard in the initial period, 
$t=0$, but exerting no effort in the subsequent $T-1$ periods, $t=1,2, \ldots, T-1$. The non-negativity constraint on capital binds only from period $t=-1$ to $t=0$ and from period $T-1$ to $T$ and so on. In the other periods, $t=0,1, \ldots, T-2$, consumption follows the Euler-equation path, equation (A.9).

Let $\nu_{T}\left(k_{0}, k_{T}\right)$ be the value to a $T$-period cycle when the planner begins with initial capital $k_{0}$ but must bequeath capital $k_{T}$ at the end of the cycle:

$$
\begin{gathered}
\nu_{T}\left(k_{0}, k_{T}\right)=\max _{n, c_{0}, \ldots, c_{T-1}}\left\{\sum_{t=0}^{T-1} \beta^{t} \log \left(c_{t}\right)\right\}-\phi n, \text { subject to: } \\
k_{1}=(1-\delta) k_{0}-c_{0}+n^{1+\gamma}, k_{t+1}=(1-\delta) k_{t}-c_{t}, t=1, \ldots, T-1 .
\end{gathered}
$$

This problem can be rewritten as:

$$
\nu_{T}\left(k_{0}, k_{T}\right)=\max _{n} m \log \left[\frac{1}{m}\left(n^{1+\gamma}+(1-\delta) k_{0}-\frac{k_{T}}{(1-\delta)^{T-1}}\right)\right]-\phi n+M
$$

Here:

$$
\begin{aligned}
& m=\frac{1-\beta^{T}}{1-\beta} \\
& M=\log ((1-\delta) \beta)\left\{\frac{\beta-\beta^{T}}{(1-\beta)^{2}}-\frac{T-1}{1-\beta} \beta^{T}\right\} .
\end{aligned}
$$

The planner's choice of $n$, given $T$, pins down initial consumption, $c_{0}$; the Euler equation (A.9) pins down the subsequent trajectory of consumption for the balance of the cycle.

Note that the constant-labor policy is just equal to the value of pursuing a one-period cycle endlessly, $\bar{v}=(1 /(1-\beta)) \nu_{1}(0,0)$. With this in mind, we can compute the value of pursuing a two-period cycle endlessly; $1 /(1-\beta) \nu_{2}(0,0)$. It turns out that:

$$
\begin{aligned}
& \nu_{1}(0,0)=(1+\gamma)\left[\log \left(\frac{1+\gamma}{\phi}\right)-1\right], \\
& \nu_{2}(0,0)=(1+\beta)(1+\gamma)\left[\log \left(\frac{1+\gamma}{\phi}\right)-1\right]+M
\end{aligned}
$$


The associated value functions are $\nu_{1} /(1-\beta)$ for the constant-effort policy, and $\nu_{2} /\left(1-\beta^{2}\right)$ for the two-period cycle. For the two period cycle to provide more utility than the constant-effort case, it must be the case that:

$$
\gamma \geq \frac{\beta}{1+\beta} \frac{\log \left[((1-\delta) \beta)^{-1}\right]}{\log (1+\beta)}
$$

This is exactly our definition of $\gamma^{\star}$ from the theorem. Thus we know that when $\gamma \geq \gamma^{\star}$ some form of cycle will be optimal.

Let $V(k)$ be the fixed point of $\mathcal{T}$ as before; also let $c(k)$ and $n(k)$ denote the optimal consumption and effort policies associated with $V(k)$.

We now argue that:

$$
V(k)=\max _{T}\left\{\nu_{T}(k, 0)+\beta \frac{\nu_{T}(0,0)}{1-\beta^{T}}\right\} .
$$

Consider the case when $\gamma=\gamma^{\star}$. As we saw, a two-period cycle provided more utility than the constant-labor policy. Could the planner do better than a twocycle? The only other possibility is that the planner work some positive amount each period. If that is the case, then the non-negativity constraints on capital never bind, and from the sequence problem (SP) above:

$$
n_{t+1}=[(1-\delta) \beta]^{1 / \gamma} n_{t}
$$

However, because $(1-\delta) \beta<1$ and $\gamma>0$, this implies that $n_{t+1}<n_{t}$, or that consumption falls smoothly over time. This in turn implies that at some period $\tau$ in the future, no matter how large $n_{0}$ is, $n_{\tau}<\gamma / \phi$. This is contradiction of lemma 2.3, which holds that effort is either zero or greater than $\gamma / \phi$. Thus the non-negativity constraints must bind sometime. If they do not bind every period, the planner works some amount and consumption is guided by the unconstrained Euler equation thereafter, until the non-negativity constraint binds again.

Consider the policy associated with a $T$-cycle. When initial capital is zero, the planner works and consumes $n_{0}$ and $c_{0}$ given by:

$$
\begin{aligned}
& n_{0}=m(T) \frac{1+\gamma}{\phi} \\
& c_{0}=\frac{1}{m(T)} n_{0}^{1+\gamma}
\end{aligned}
$$


We can then derive the end-of-period capital stock, $k_{1}$, associated with this policy. After substituting back in for the constant term $m(T)$ we find that:

$$
k_{1}=\left(\frac{\beta-\beta^{T}}{1-\beta^{T}}\right)\left(\frac{1-\beta^{T}}{1-\beta} \frac{1+\gamma}{\phi}\right)^{1+\gamma} .
$$

In the same way, we can find the penultimate positive capital stock, $k_{T-1}$ :

$$
\frac{k_{T-1}}{(1-\delta)^{T-2}}=\left(\frac{\beta^{T-1}-\beta^{T}}{1-\beta^{T}}\right)\left(\frac{1-\beta^{T}}{1-\beta} \frac{1+\gamma}{\phi}\right)^{1+\gamma} \text {. }
$$

Denote this capital stock $\bar{k}(T)$; for all capital $k>\bar{k}(T)$ the planner's optimal policy is to consume a portion of the capital stock and exert zero effort. At some capital stock $0<\kappa<\bar{k}(T)$, the planner begins again to work. For capital stocks $\bar{k}(T)>k>\kappa$ the planner's optimal policy is to exert no effort and consume the capital stock entirely, setting $k^{\prime}=0$. For capital sotcks $k<\kappa$ the planner's optimal policy is to work hard and set $k^{\prime} \geq \bar{k}(T)$. By the envelope theorem we know that, for some small $\epsilon>0$ :

$$
\begin{aligned}
& \left.\frac{d}{d k} V(k)\right|_{k=\kappa+\epsilon}=\frac{\partial F(k, 0)}{\partial k}, \\
& \left.\frac{d}{d k} V(k)\right|_{k=\kappa-\epsilon}=\frac{\partial F\left(k, k^{\prime}\right)}{\partial k} .
\end{aligned}
$$

From lemma 4, we know, when $k^{\prime}$ is large relative to $k$ (as is the case here), that:

$$
\frac{\partial F(k, 0)}{\partial k}>\frac{\partial F\left(k, k^{\prime}\right)}{\partial k}
$$

At the higher capital stock, the planner does not work, while at the lower, he does. Hence in a local region around $\kappa$ the planner is a risk lover. Hence the planner would prefer a fair gamble with expected value $\kappa$ than $\kappa$ with certainty. 


\section{B Comparison with Christiano and Harrison}

Following section 6.2 of Christiano and Harrison, assume a social planner maximizes:

$$
\sum_{t=0}^{\infty} \beta^{t}\left[\log \left(c_{t}\right)+\sigma \log \left(1-n_{t}\right)\right]
$$

where $\sigma>0$ and $c_{t}, n_{t} \geq 0$. The law of motion for capital is:

$$
k_{t+1} \leq\left(k_{t}\right)^{\alpha}\left(n_{t}\right)^{2}+(1-\delta) k_{t}-c_{t}
$$

and $k_{0}$ is given. We generalize $\mathrm{CH}$ by allowing for either diminishing or constant returns to capital $0 \leq \alpha \leq 1$.

Under constant returns to capital $\alpha=1, \mathrm{CH}$ show that the optimal labor supply is constant. It is useful to review $\mathrm{CH}$ 's derivation of the optimal allocation. Without satiation, equation (B.2) holds with equality and we use it to substitute $c_{t}$ out of the objective, obtaining:

$$
\sum_{t=0}^{\infty} \beta^{t}\left\{\log \left[k_{t}\left(n_{t}\right)^{2}+(1-\delta) k_{t}-k_{t+1}\right]+\sigma \log \left(1-n_{t}\right)\right\} .
$$

Defining $\lambda_{t}$ as the gross growth rate of capital, $k_{t+1} / k_{t}$, this expression becomes:

$$
\sum_{t=0}^{\infty} \beta^{t}\left\{\log \left(k_{t}\right)+\log \left[\left(n_{t}\right)^{2}+(1-\delta)-\lambda_{t}\right]+\sigma \log \left(1-n_{t}\right)\right\} .
$$

$\mathrm{CH}$ show that the discounted sum of the log capital stock can be expressed as a function of $k_{0}$ and the $\left\{\lambda_{t}\right\}_{t=0}^{\infty}$ sequence: $^{5}$

$$
\sum_{t=0}^{\infty} \beta^{t} \log \left(k_{t}\right)=\frac{1}{1-\beta} \log \left(k_{0}\right)+\frac{\beta}{1-\beta} \sum_{t=0}^{\infty} \beta^{t} \log \left(\lambda_{t}\right) .
$$

\footnotetext{
${ }^{5}$ Rearranging the elements of an infinite sum requires the sum to be well defined for appropriate sequences, which holds in this case.
} 
Substituting (B.5) into (B.4), the social planner's objective becomes:

$$
\begin{aligned}
& \frac{1}{1-\beta} \log \left(k_{0}\right)+\sum_{t=0}^{\infty} \beta^{t}\left\{\operatorname { l o g } \left[\left(n_{t}\right)^{2}+\right.\right. \\
& \left.\left.\quad(1-\delta)-\lambda_{t}\right]+\frac{\beta}{1-\beta} \log \left(\lambda_{t}\right)+\sigma \log \left(1-n_{t}\right)\right\}
\end{aligned}
$$

subject to $n_{t} \geq 0$. $^{6}$

The dynamic problem in equation (B.6) can be converted into a sequence of static problems. Begin by noticing that consumption in period $t$ can be written as a product of the preceding values of capital growth $\lambda_{t}$, the initial capital stock $k_{0}$, and the current consumption-capital ratio, $c_{t} / k_{t}$ :

$$
\begin{aligned}
c_{t} & =\left(\frac{c_{t}}{k_{t}}\right) k_{t}, \\
& =\left(\frac{c_{t}}{k_{t}}\right) \lambda_{t-1} \lambda_{t-2} \cdots \lambda_{0} k_{0} .
\end{aligned}
$$

Use the budget constraint (B.2) to write consumption $c_{t}$ as a function of current and future capital, $k_{t}, k_{t+1}$. Substituting produces:

$$
c_{t}=\left[\left(n_{t}\right)^{2}+1-\delta-\lambda_{t}\right] \lambda_{t-1} \lambda_{t-2} \cdots \lambda_{0} k_{0} .
$$

The time zero welfare contribution of consumption at time $t$ is then:

$$
\beta^{t} \log \left(c_{t}\right)=\beta^{t}\left(\log \left(k_{0}\right)+\log \left[\left(n_{t}\right)^{2}+1-\delta-\lambda_{t}\right]+\sum_{j=0}^{t-1} \log \left(\lambda_{t}\right)\right)
$$

The separability between differently dated choice variables in (B.6) is a result of constant returns to capital in production and log preferences over consumption.

We modify $\mathrm{CH}$ by assuming diminishing instead of constant returns to capital. $\mathrm{CH}$ find this assumption useful, in part, because it delivers a closed form solution for the optimal resource allocation. Linear production in capital is often used in endogenous-growth models; by contrast, business cycle modelling in an animal

\footnotetext{
${ }^{6}$ Bounds on $\lambda_{t}$, which reflect the requirement that $c_{t}, k_{t} \geq 0$, never bind because of Inada conditions.
} 
spirits context more commonly uses decreasing returns to capital. ${ }^{7}$

Intuitively, if production is linear in capital, an oscillating path for labor is less likely to be optimal. Suppose the planner is trying to improve on a constant labor supply allocation by taking advantage of increasing returns to labor. Imagine that, instead of supplying the same amount of labor for two periods in a row, the planner increases labor supply this period in order to finance consumption both this period and next, when the planner decreases labor supply. By concentrating labor effort in the first period, the planner takes advantage of increasing returns to scale, achieving the same output (in present value) at lower utility cost (again, in present value). However, the capital stored by the planner this period (in order to finance consumption next period) increases the marginal productivity of labor next period. Moreover, this increase is always independent of the current level of the capital stock, because production is linear in capital. Thus, with the higher labor productivity in the next period, the planner faces an additional incentive to work. ${ }^{8}$ Without diminishing returns to capital, the effect on the productivity of future labor grows linearly as greater savings is undertaken.

Let us continue characterizing the optimal allocation when $\alpha=1$. The planner chooses $\lambda_{t}=\lambda, n_{t}=n$ for all $t \geq 0$. to maximize:

$$
\log \left(n^{2}+1-\delta-\lambda\right)+\frac{\beta}{1-\beta} \log (\lambda)+\sigma \log (1-n),
$$

subject to $n \geq 0$. CH show that, given $n$, the problem is strictly concave in $\lambda$, and concentrate the objective function into solely a function of $n$ :

$$
L(n)=\frac{1}{1-\beta} \log \left(n^{2}+1-\delta\right)+\sigma \log (1-n) .
$$

\footnotetext{
${ }^{7}$ As an example, most of the papers we cite in our introduction use models with diminishing returns to capital.

${ }^{8}$ This argument may seem to suggest that the optimal resource allocation involves infinite labor supply and utility. There are two things preventing this. First, $\mathrm{CH}$ households face high utility penalties as labor grows-which bounds the amount of labor effort in the presence of arbitrary increasing returns. Second, the discrete time formulation also limits the usefulness of extremely high labor allocations. In essence, the household would like to work very hard for a very brief period of time, but the discrete-time formulation forces the household to choose its labor effort choice for an entire period.
} 
Figure 16 plots the function $L$. The local maximum is reached at $\tilde{n}$, where:

$$
\tilde{n}=\frac{1}{2}\left[\Phi+\sqrt{\Phi^{2}-4 \xi}\right], \Phi=\frac{2}{2+\sigma(1-\beta)}, \xi=\frac{\sigma(1-\beta)(1-\delta)}{2+\sigma(1-\beta)}
$$

The globally optimal labor input is time invariant, independent of the capital stock and equals either $n=0$ or $n=\tilde{n}$.

Figure 16 is drawn so that $L(\tilde{n})>L(0)$. This inequality may be reversed for some parameterizations, in which case not working is optimal. In the zero labor optimal allocation, the household consumes a fraction of the undepreciated capital stock each period. In the neoclassical model, Inada conditions typically rule out the optimality of zero labor; however, with preferences (B.1) and resource constraint (B.2), the marginal product of labor is zero and the marginal disutility of work is finite at $n=0$.

Next, we seek to show that it is possible for $L(\tilde{n})<L(0)$. Let $G=L(\tilde{n})-$ $L(0)$. If we define $x=1-\delta, z=1-\beta$, then $G$ is given by:

$$
G(x, z, \sigma)=z^{-1} \log \left[\left(\frac{\tilde{n}}{\sqrt{x}}\right)^{2}+1\right]+\sigma \log (1-\tilde{n})
$$

where $\tilde{n}$ is defined by (B.7). Using the envelope theorem, $\partial G / \partial x<0$. An increase in the retention rate makes it more likely that $L(\tilde{n})<L(0)$ and zero labor is optimal. The maximum value we can select for $x$, and still have real-valued $\tilde{n}$ and $0 \leq \delta<1$, is $x^{\star}(z, \sigma)=\min \{1 /[\sigma z(2+\sigma z)], 1\}$.

If $\sigma z(2+\sigma z)>1$, then:

$$
\tilde{n}=\frac{1}{2+\sigma z}
$$

In this case:

$$
\begin{gathered}
G\left[x^{\star}(z, \sigma), z, \sigma\right]<0 \text { iff: } \\
\log \left[\frac{2(1+\sigma z)}{2+\sigma z}\right]<\sigma z \log \left(\frac{2+\sigma z}{1+\sigma z}\right)
\end{gathered}
$$

To illustrate the possibility of zero labor being optimal, assume $\sigma z=1$. Then $G<0$ since $\log (4 / 3)<\log (3 / 2)$.

The optimality of such a corner solution is more likely for larger values of $\sigma$, which implies a greater disutility of work, and for lower values of $\delta$, which 
increases the usefulness of capital for storage.

Next, consider the case of diminishing returns to capital. Since the model no longer admits a closed form solution, we take a stand on parameter values. The parameters $(\sigma, \beta, \delta)=\left(2.0,1.03^{-1 / 4}, 0.02\right)$ match those chosen by $\mathrm{CH}$. Whereas $\mathrm{CH}$ choose $\alpha=1$, we set $\alpha=1 / 3$. We compute the optimal solution by discrete discounted dynamic programming on a finite capital grid using value function iteration.

Figure 17 plots the optimal policy functions for investment (next period capital), labor and consumption as a function of the current period capital stock. Note the discontinuous drop in labor at approximately $\tilde{k} \approx 6.2$. At low capital levels, labor input is high in order to increase the capital stock, which increases the future marginal product of labor. For sufficiently high levels of capital $k>\tilde{k}$, however, labor input is set equal to zero. Because of diminishing returns to capital, it is costly in terms of labor effort to produce the output necessary to maintain a high capital stock. Instead, the social planner halts production and allows the capital stock to decline due to depreciation and the consumption of existing capital.

Next, note the associated discontinuity in the capital policy, which occurs at the same capital level as the labor policy. For $k<k$, the capital policy function lies above the 45 degree line; hence, the social planner engages in net investment. For any $0<k_{0}<\tilde{k}$, in a finite number of periods the capital stock will leave the $(0, \tilde{k})$ region for the first, but not the last time. Once the capital stock is greater than $\tilde{k}$, the capital policy function lies below the 45 degree line. Instead of net investment, there is disinvestment. In this region, since labor is zero, any consumption comes from undepreciated capital. For this reason as well as standard depreciation, the capital stock falls. High levels of the capital stock are expensive to maintain in terms of labor effort and the social planner finds it optimal to shut down production and consume part of the capital stock. Eventually, the capital stock falls enough to return to the $(0, \tilde{k})$ region.

The optimal capital policy exhibits endogenous cycles with periods of positive net investment and high labor input, each of which is followed by declining capital and zero labor. These cycles are displayed in figure 18 below. The policy of production bunching in the presence of increasing returns to labor is intuitive: intense labor supply - when the marginal product of labor is high-is followed by a period of labor inactivity, as is the case in Murphy, Shleifer and Vishny (1989). There are other, slight discontinuities in the capital policy function besides that at $k=\tilde{k}$, although they are more difficult to see.

The consumption plan associated with the optimal investment and labor policies requires that the planner eats part of the existing capital stock. The optimal 
allocation involving labor cycles requires the household to consume out of the undepreciated capital stock in periods where labor equals zero. This, in turn, requires that the household transform capital back into consumption goods. That is, investment must be reversible. If investment were irreversible, by contrast, zero labor would not be optimal because consumption would be zero (and marginal utility infinite) in those periods. One interpretation for this reversibility is that the capital stock includes final goods inventories, as well as equipment and structures.

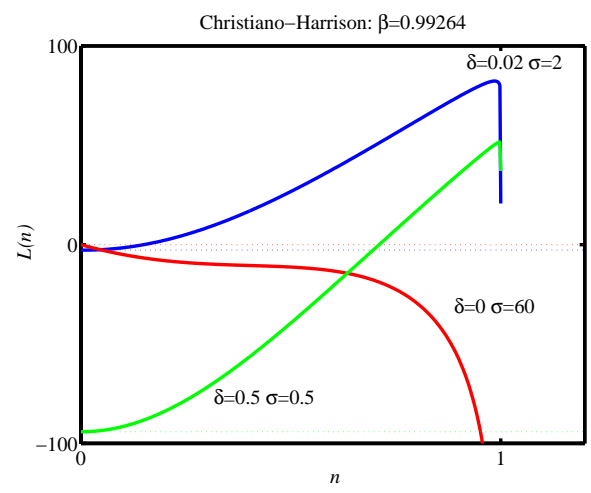

Figure 16: Function $L(n)$ for various parameter combinations. The dashed horizontal lines give $L(0)$ for each parameter combination.

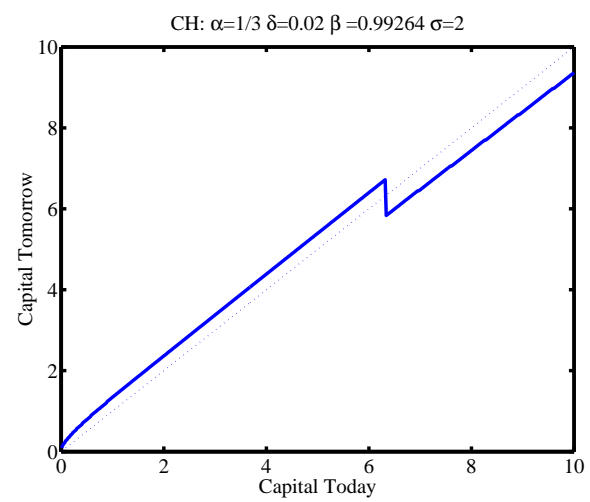

a. Investment policy

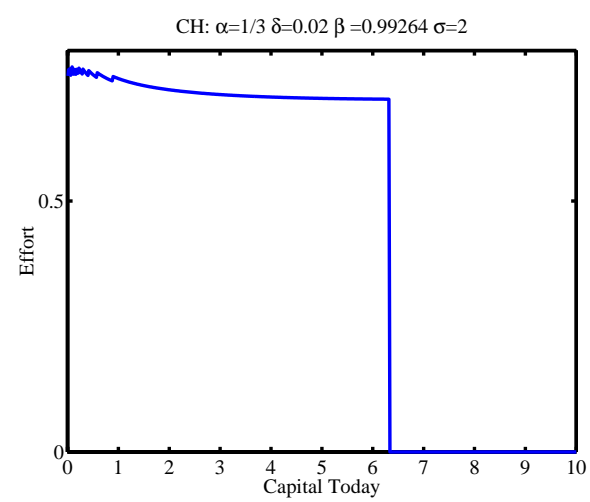

b. Labor policy

Figure 17: Christiano and Harrison model with $\alpha<1$. 

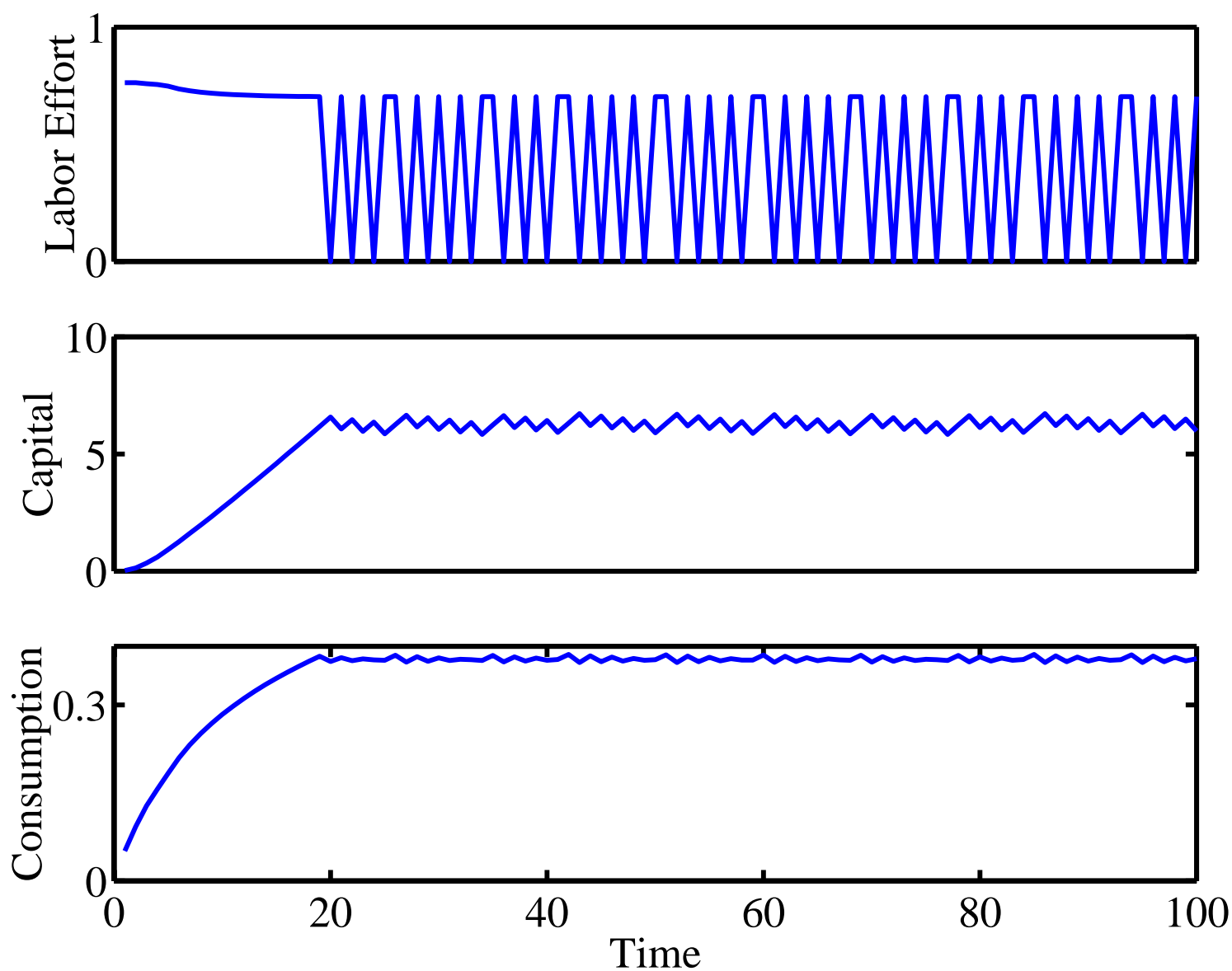

Figure 18: Dynamics in the Christiano and Harrison model with $\alpha<1$. 


\section{References}

Baxter, M. and R. G. King (1991). Productive externalities and business cycles. Institute for Empirical Macroeconomics Discussion Paper 53, Federal Reserve Bank of Minneapolis.

Benhabib, J. and R. Farmer (1994). Indeterminacy and increasing returns. Journal of Economic Theory 63(1), 19-41.

Benhabib, J. and R. Farmer (1996). Indeterminacy and sector-specific externalities. Journal of Monetary Economics 37(3), 421-443.

Blinder, A. A. and L. J. Maccini (1991). Taking stock: A critical assessment of recent research on inventories. Journal of Economic Perspectives 5(1), 73-96.

Boldrin, M. and A. Rustichini (1994). Growth and indeterminacy in dynamic models with externalities. Econometrica 62(2), 323-342.

Christiano, L. J. and S. G. Harrison (1999). Chaos, sunspots, and automatic stabilizers. Journal of Monetary Economics 44(1), 3-31.

Farmer, R. and J.-T. Guo (1994). Real business cycles and the animal spirits hypothesis. Journal of Economic Theory 63(1), 42-72.

Farmer, R. and J.-T. Guo (1995). The econometrics of indeterminacy: An applied study. Carnegie-Rochester Conference Series on Public Policy 43(0), 225-271.

Guo, J.-T. and K. Lansing (1998). Indeterminacy and stabilization policy. Journal of Economic Theory 82(2), 481-490.

Guo, J.-T. and K. Lansing (2001). Fiscal policy, increasing returns and endogenous fluctuations. Forthcoming in Macroeconomic Dynamics.

Judd, K. L. (1999). Numerical Methods in Economics. Cambridge, MA: The MIT Press.

Krugman, P. (1999). The role of geography in development. International Regional Science Review 22(2), 142-61.

Lehnert, A. (1998). Asset pooling, credit rationing, and growth. Finance and Economics Discussion Series 1998-52, Federal Reserve Board.

Lucas, Jr., R. E. (1993). Making a miracle. Econometrica 61(2), 251-272. 
Murphy, K. M., R. W. Vishny, and A. Shleifer (1989a). Building blocks of market-clearing business cycle models. In NBER Macroeconomics Annual, pp. 247-287. Cambridge, MA: MIT Press.

Murphy, K. M., R. W. Vishny, and A. Shleifer (1989b). Industrialization and the big push. Journal of Political Economy 97(5), 1003-1026.

Paulson, A. L. and R. Townsend (2000, November). Entrepreneurship and financial constraints in Thailand. Manuscript, University of Chicago.

Phelan, C. and R. M. Townsend (1991). Computing multi-period, informationconstrained optima. Review of Economic Studies 58(5), 853-882.

Pontryagin, L., V. Boltyanski, R. Gamkrelidze, and E. F. Mishchenko (1964). The Mathematical Theory of Optimal Processes. New York, NY: Pergamon Press.

Prescott, E. and R. Townsend (1984a). General competitive analysis in an economy with private information. International Economic Review 25(1), 1-20.

Prescott, E. and R. Townsend (1984b). Pareto optima and competitive equilibria with adverse selection and moral hazard. Econometrica 52(1), 21-45.

Romer, P. (1986). Cake eating, chattering, and jumps: Existence results for variational problems. Econometrica 54(4), 897-908.

Stokey, N. L. (1981). Rational expectations and durable goods pricing. Bell Journal of Economics 12(1), 112-128.

Stokey, N. L. and R. E. Lucas, Jr. (1989). Recursive Methods in Economic Dynamics. Harvard University Press.

Subrahmanyam, A. and S. Titman (1999). Real effects of financial market trading: Market crises and the going public process. Manuscript, College of Business Administration, University of Texas at Austin. 